Revue

de l'histoire des religions

\section{Revue de l'histoire des religions}

$4 \mid 2013$

Sermo mysticus Mystique et langage entre Moyen Âge et époque moderne

\title{
La plume et l'envol : une analyse stylistique de la Méditation sur l'Ascension de Jean Gerson
}

The feather and the flight: a stylistic analysis of the Meditation on the Ascension by Jean Gerson

Isabelle Fabre

\section{OpenEdition}

\section{Journals}

Édition électronique

URL : http://journals.openedition.org/rhr/8175

DOI : $10.4000 /$ rhr.8175

ISSN : 2105-2573

Éditeur

Armand Colin

\section{Édition imprimée}

Date de publication : 1 décembre 2013

Pagination : 509-544

ISBN : 978-2200928667

ISSN : 0035-1423

Référence électronique

Isabelle Fabre, «La plume et l'envol : une analyse stylistique de la Méditation sur l'Ascension de Jean Gerson », Revue de l'histoire des religions [En ligne], 4 | 2013, mis en ligne le 01 décembre 2016, consulté le 04 mai 2019. URL : http://journals.openedition.org/rhr/8175; DOI : 10.4000/rhr.8175 


\section{ISABELLE FABRE}

Université de Montpellier 3

\section{La plume et l'envol : une analyse stylistique de la Méditation sur l'Ascension de Jean Gerson}

Sous les dehors d'un opuscule de dévotion ascétique, la Méditation sur l'Ascension de Jean Gerson (1363-1429) est un texte hybride qui exploite les contrastes stylistiques pour suggérer le progrès dans la "contemplation». Le parcours ascensionnel n'est pas seulement visualisé par l'image de l'oiseau s'élevant dans les cieux; il se construit aussi par les métamorphoses successives de l'écriture, en trois étapes qui correspondent d'une part aux degrés de l'échelle spirituelle, de l'autre à trois techniques de compositio assignables à trois « genres»: la méditation affective, le sermon de tempore et le traité de théologie mystique. On montrera comment ces catégories, subtilement articulées les unes aux autres, donnent au texte son rythme spécifique, en même temps qu'une profondeur doctrinale.

The feather and the flight: a stylistic analysis of the Meditation on the Ascension by Jean Gerson

While it resembles a short text of ascetic devotion, the Meditation on the Ascension by Jean Gerson contains some hybrid features and draws on stylistic contrasts to suggest the progress of the soul in "contemplation". The ascending process is not only portrayed through the image of a bird flying up to the skies; it also emerges through the metamorphoses of writing, in three stages which correspond on the one hand to the degrees of the spiritual ladder, on the other hand to three modes of composition that can be associated with several genres: affective meditation, the de tempore sermon and the treatise on mystical theology. I shall make clear how these categories are subtly interconnected with one another, giving the whole text its peculiar rhythm, along with a genuine doctrinal complexity. 
Le discours mystique peint souvent son objet en termes d'élévation ou d'ascension spirituelle. Mais tout discours d'élévation n'est pas mystique pour autant. La frontière est mince entre les différentes modalités de l'expérience spirituelle et les manières d'en rendre compte à l'écrit. Il est des œuvres qui semblent brouiller à dessein les frontières et qui, tout en s'inscrivant dans une tradition mystique bien attestée, semblent par trop maîtrisées pour ne pas appeler une lecture distanciée. Leur but est autre. Il s'agit moins de dire l'ineffable, ou d'exprimer les effets de la grâce, que de susciter la ferveur par une image anagogique, celle de l'envol. Le texte de Gerson qu'on se propose d'éclairer est de ceux-là. Chancelier de l'Université de Paris, théologien et prédicateur particulièrement actif durant le Concile de Constance, Jean Gerson (1363-1429) laisse une œuvre abondante, tant en latin qu'en français, où dominent deux préoccupations : la réforme de l'Université, à travers l'enseignement de la théologie mystique, et l'éducation chrétienne des «simples gens $»^{1}$. La Méditation sur l'Ascension s'inscrit dans cette double démarche, très productive à partir des années 1400 . Opuscule n'ayant semble-t-il connu qu'une faible diffusion, copié dans deux recueils aux côtés d'autres petits traités de spiritualité et de sermons anonymes ou attribués à Gerson ${ }^{2}$, la Méditation sur l'Ascension ne relève pas en apparence du sermo mysticus. Son objet est, plus modestement, la pédagogie de la vie intérieure. Mais elle mobilise des images et des figures qui invitent à une lecture plus ambitieuse, où se dessine en filigrane un parcours ascensionnel débouchant sur les sommets de la contemplation. Par-delà une topique bien connue, d'origine à la fois biblique et platonicienne ${ }^{3}$,

1. Pour une présentation détaillée de la carrière et de l'œuvre du Chancelier, voir la récente biographie de Brian Patrick McGuire, Jean Gerson and the Last Medieval Reformation, State College, The Pennsylvania State University Press, 2005.

2. BnF, fr. 990 et fr. 24839. Voir l'introduction à notre édition en annexe de la présente étude. Ce type de compilation est fréquent au $\mathrm{XV}^{\mathrm{e}}$ siècle, même si les recueils d'auteur s'avèrent plus nombreux (voir Geneviève Hasenohr, «Aperçu sur la diffusion et la réception de la littérature de spiritualité en langue française au dernier siècle du Moyen Âge», Wissensorganisierende und wissensvermittelnde Literatur im Mittelalter, éd. Norbert Richard Wolf, Wiesbaden, Dr. Ludwig Reichert Verlag, 1987, p. 75-76).

3. Platon, Phèdre, 246a-256e (trad. Émile Chambry, Paris, GF-Flammarion, 
celle de l'envol de l'âme, nous montrerons comment l'auteur fabrique un texte hybride. Son originalité formelle tient à ce qu'il mime, par les métamorphoses successives de son écriture, un processus ascensionnel qui figure l'approfondissement de la méditation. En nous appuyant sur les outils de l'analyse stylistique, nous soulignerons la structure tripartite du texte qui correspond, d'une part, aux trois degrés traditionnels de l'échelle spirituelle et, de l'autre, à trois techniques de compositio que nous référerons à trois «genres» au sein de la littérature d'édification: la méditation affective, le sermon et le traité didactique. On mettra en évidence les principes poétiques sur lesquels repose le montage gersonien, en suivant pas à pas ses étapes et en les rapportant à leurs sources littéraires et théologiques, afin de montrer comment ils confèrent au texte son unité sous-jacente, ainsi qu'une dynamique qui tend à reléguer au second plan l'aspect décousu de la composition. À cette fin, on s'appuiera sur une version neuve du texte, présentée en annexe intégralement revue et corrigée, et accompagnée d'un double apparat de notes visant à restituer de manière aussi précise que possible sa richesse philologique et la variété de ses modèles et registres.

\section{$1^{\text {er }}$ DEGRÉ : LA DIFFICULTÉ DE L'ENVOL \\ - DE L'ORATIO VÉHÉMENTE À LA MEDITATIO EFFUSIVE}

«Qui me dourra pennes et elles a la semblance d'une colombe? Si voleray et me reposeray.» Le verset du Ps. 54 sur lequel s'ouvre le texte en donne la clé en même temps que la tonalité. Il exprime un désir à deux facettes antithétiques, désir d'élan et de repos. Il dit aussi le désarroi d'une question lancée dans le vide, sans certitude de retour. Désir et espoir d'un côté, plainte et déréliction de l'autre: ce thema scripturaire inaugure l'étape purgative, point de départ de l'examen de soi. Mais il faut entendre l'appel du Psalmiste sur la toile de fond néotestamentaire, car la question est aussi la réponse à un événement précis; le désir d'envol surgit d'une envolée première, celle du Christ lors de son Ascension:

1964, p. 125). L'histoire du motif dans sa double tradition est longuement étudiée par Pierre Courcelle, "Connais-toi toi-même » de Socrate à saint Bernard, t. 2***, ch. XVI, 3 («L'envol de l'âme»), Paris, Études augustiniennes, 1975, p. 562-623. 
Veez cy que mon Dieu, mon maistre et mon espoux s'en vole en hault, plus hault que les vens dessus toutes les nues, voir au dessus de tous les cieulx; et je, povre ame desolee, demeure en bas, trop bas sa jus en terre: comme l'ensuivray je ?

La scène qui clôt l'Évangile de Luc (Lc 54, 50-53) marque la fin du ministère de Jésus et son adieu à ses disciples. La méditation place le je dans la contemplation d'un envol et la déploration d'une perte; elle rythme de son flux et reflux la tension contradictoire qui sera celle de tout le texte: au vocatif ternaire ( «mon Dieu, mon maistre et mon espoux») et à la gradatio, ternaire elle aussi («en hault, plus hault... voir au dessus ») qui emportent le Christ jusqu'à l'acmé des cieux, répond le versant descendant de la phrase («en bas, trop bas»), amputé d'un élément pour mieux rendre l'effet, l'abattement de l'âme. L'anaphore et les parallélismes syntaxiques renforcent ce sentiment d'accablement, tandis que l'alternance des questions et des exhortations, jointe à l'intrusion du dialogisme par lequel l'âme se prend elle-même à partie, traduit l'instabilité d'un état oscillant entre prostration et sursauts de vigueur morale:

Je suis lasse orpheninne; je suis veve desconfortee: // qui sera mon tuteur? Qui me consolera? // Apreste toy a voler, a ensuir ton amy. // Tu triste de cuer, reprens couraige, efforce toy ${ }^{5}$ !

L'échec de cet envol initial marque le début de la méditation introspective. Elle prend pour matière l'image de l'oiseau, dont les traits définitoires (plumes et ailes) font l'objet d'une interprétation allégorique: les plumes sont de «sainctes pensees », les deux ailes, «l'une de bon desir, l'autre de devot espoir». La symbolique n'est pas nouvelle. Elle prolifère depuis les Pères, qui multiplient les correspondances avec les bonnes œuvres (jeûne et aumône ${ }^{6}$ ) et les vertus (humilité et obéissance, charité et pudeur, etc. ${ }^{7}$ ), quand

4. Méditation sur l'Ascension, § 2. Les citations réfèrent à notre édition en annexe.

5. Ibid., $\$ 2$.

6. Augustin, Sermo 206, 2 (PL 38, col. 1041-1042) et Enarrationes in Psalmos LXII-8 (éd. Eligius Dekkers et Jean Fraipont, Turnhout, Brepols, CCSL 38, $2^{e}$ éd., 1990, p. 481, 1. 19-20).

7. Césaire d'Arles, Sermo 234, 2 (éd. Germain Morin, Turnhout, Brepols, Corpus Christianorum Series Latina 104, 1953, § 887, p. 933): «si istas duas virtutum alas, id est humilitatem et obeodientiam non habuerit, in altum evolare non poterit». Léon le Grand, Sermo LV, 5 (PL 54, col. 325): «charitatis opere ac nitore pudicitiae, tamquam duabus elevati alis ». 
ils n'y voient pas un lien avec la Règle d'or (amour de Dieu et du prochain) $)^{8}$. Gerson lui-même ne se fait pas faute d'exploiter longuement l'image dans la considération 11 de son traité $D e$ theologia mystica practica: chaque aile comprend dix plumes principales (pennae) fractionnables à leur tour en diverses petites plumes (pennulae) qui réfèrent à des «méditations particulières» sur la crainte (aile de gauche) et l'amour de Dieu (aile de droite) ${ }^{9}$. Mais on n'est pas ici dans le cadre de l'industria méthodique ${ }^{10}$, qui se développe systématiquement sub typo columbe, dans un but mnémonique et agence ses détails en les référant à différentes «matières» dans un ordonnancement hiérarchique laissé pour une part ad libitum. L'image fournit un ancrage à la méditation, sans pour autant que celle-ci s'y réduise.

Elle est donc polyvalente. On en trouve une exploitation assez proche dans le Jardin amoureux de l'âme de Pierre d'Ailly:

Ce font les oyseillons qui de terre volent au ciel en ostant les plumes de leurs cogitations hors mondaine occupation et en mouvant les ailes de leurs affections par divines meditations. Ainsi volent legierement et montent haultement les ames devotes ${ }^{11}$.

Mais ce qui, chez le maître de Gerson, exprime le passage réussi de la vie active à la vie contemplative, est ici pris à rebours. Après une nouvelle plainte interrogative, le revirement s'opère, par l'image de l'oiseau pris dans la matière, et qui se déplume et s'englue:

Mais helas, que sont devenus mes plumes de sainctes pensees et mes deux elles, l'une de bon desir, l'autre de devot espoir? Elles sont esparses ça et la en maintes parties, par evagacion de mon cueur en

8. Chez Augustin, Enarr. in Ps. CIII, sermo I, 12-13 (CCSL 40, p. 14851486, 1. 31-33) et Césaire d'Arles, Sermo 82, 2 (éd. G. Morin, CCSL 103, § 323, p. 337-338). Pour un récapitulatif de ces différentes lectures, voir P. Courcelle, op. cit., p. 613-615.

9. Voir le texte latin dans l'édition critique d'André Combes, Ioannis Carlerii de Gerson De mystica theologia, Lugano, Thesaurus mundi, 1958, p. 203-207.

10. Gerson nomme de ce terme un type d'exercice spirituel fondé sur une image allégorique à valeur mnémonique.

11. Le texte est édité parmi les œuvres de Gerson par Palémon Glorieux (Jean Gerson, Euvres complètes, t. VII, Paris, Desclée \& Cie, 1966, n 309, p. 151). Sur l'attribution à Pierre d'Ailly, voir l'étude de Pierre-Yves Badel, «Pierre d'Ailly auteur du Jardin Amoureux», Romania, XCVII, 1976, p. 369-381. Pour une étude du motif replacé dans son cadre allégorique, je me permets de renvoyer à mon article, «Aspects de l'allégorie spirituelle au $\mathrm{Xv}^{\mathrm{e}}$ siècle: Le Jardin amoureux de l'âme de Pierre d'Ailly », Des pouvoirs visionnaires de l'allégorie, éd. Frédérique Toudoire-Surlapierre et Nicolas Surlapierre, Paris, L'improviste, 2012, p. 45-65. 
cures terriennes, et ce peu qui en est demouré, qui n'est point du tout desraciné, helas que me prouffite? La tenant de charnalité corrumpue a tout englué; la pesanteur de mon corps et le grief fais de mon iniquité, et me traient a terre et en si parfont que ne me puis aidier ne relever: comment voleray je ? ${ }^{12}$

Si l'oiseau est, depuis Platon et les Prophètes ${ }^{13}$, l'image-type du contemplatif attiré par les mystères divins ou l'intelligible, la métaphore se prête tout aussi facilement, dans le Phédon et dans les Psaumes, à une exploitation antithétique ${ }^{14}$. À l'aspiration pour les choses célestes, à l'élan du désir de Dieu contrevient la difficulté à s'arracher au sensible. Deux mouvements se conjuguent: la dispersion («evagacion») du cœur et la pesanteur de la chair («charnalité corrumpue»). Ces deux effets s'articulent aussi sur le plan stylistique, selon un double mécanisme d'emboîtement («ce peu qui en est demouré, qui n'est point du tout desraciné») et d'amplification polysyndétique ( et me traient a terre et en si parfont...»). Dans les deux cas, la phrase culmine et se tient en suspens sur une question rhétorique, soulignant l'irréductibilité de la chute.

La plainte alors se fait révolte et imprécation ( «maudiz soient...») car derrière la faiblesse de la créature, ce sont les «trois ennemis de l'homme» dont il s'agit de se garder. Le discours moral est traditionnel; on en trouve un traitement rhétorique similaire dans les Meditationes piissimae de cognitione humanae conditionis du Pseudo-Bernard:

Aide-moi, Seigneur mon Dieu, car mes ennemis - le corps, le monde et le diable - ont cerné mon âme. Mon corps, je ne peux le fuir, et lui non plus ne peut me fuir [...]. Le monde m'encercle et m'assiège de tous côtés et par cinq portes, c'est-à-dire par les cinq sens corporels : la vue, l'ouie, le goût, l'odorat et le toucher, il me blesse de ses flèches, et la mort pénètre en mon âme par ces fenêtres [...]. En outre, le diable, que je ne peux voir et dont je peux d'autant moins me garder, a tendu son arc et y a ajusté ses flèches, pour me blesser sans crier gare [...]. Et non seulement il a tendu son filet, mais il a posé de la glu. La glu est la passion de posséder, l'amour pour ses proches, le désir des honneurs et les plaisirs de la chair: ce sont eux qui engluent l'âme et la prennent dans leurs filets, afin qu'elle ne puisse parcourir de son vol, grâce aux ailes de la contemplation, les étendues de la Jérusalem d'en haut. Les

12. Méditation sur l'Ascension, $\S 3$.

13. Is. $38,14:$ meditabor ut columba.

14. P. Courcelle, op. cit., p. 604-612. 
flèches du diable sont la colère, l'envie, la luxure et tous les autres péchés qui blessent l'âme ${ }^{15}$.

La sobriété gersonienne est toutefois remarquable: le thème n'est pas décliné dans ses trois éléments, ni développé plus avant (pas d'arc ni de flèches allégoriques, pas de «château de l'âme»); et chez lui, «l'effet de liste» n'est pas aussi massif ni monotone. En usant de la polysyndète appliquée à un unique motif, celui du piège (« las et glut et roys et trabuchés») et en organisant l'accumulation des syntagmes au sein de la séquence par une gradation rythmique et anaphorique, Gerson accentue l'évocation de l'homme traqué sans répit par des «traitres oyselleurs» :

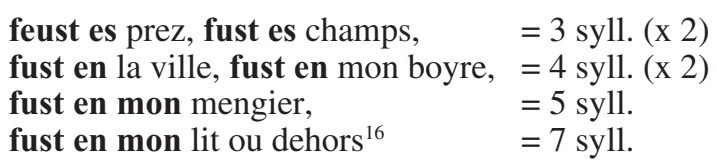

- à quoi s'ajoute un effet de parallélisme assonantique renforçant, par une cadence mineure dupliquée, la contrition de la victime, son sentiment accablant de «misere» et de «mescheance»: «Et mal me suy gardee, si suis cheute en leurs laz, et telement suis mal menee!».

Changements de dynamique et ruptures stylistiques s'ajoutent donc à la saturation métaphorique pour rendre compte du vain effort de l'âme à s'extraire de la matière. Mais ce premier envol raté met aussi en évidence un défaut de méthode: l'élévation requiert un apprentissage, et une discipline; il faut gravir l'échelle

15. Ch. 12: De tribus inimicis hominis, carne, mundo et diabolo (PL 184, col. 503-504): «Adjuva me, Domine Deus meus, quoniam inimici mei animam meam circumdederunt: corpus scilicet, mundus et diabolus. A corpore fugere non possum, nec ipsum a me fugare. [...] Mundus vero circumcingit et obsidet me undique, et per quinque portas, videlicet per quinque corporis sensus, scilicet visum, auditum, gustum, odoratum et tactum, sagittis suis me vulnerat, et mors intrat per fenestras meas in animam meam [...]. Porro diabolus quem videre non possum et ideo minus ab eo mihi cavere, tetendit arcum suum, et in eo paravit sagittas suas, ut vulneret me repente [...]. Nec suum laqueum posuit, sed et viscum. Viscus est amor possessionis, affectus cognationis, cupiditas honoris et carnis voluptas quibus anima inviscatur et irretitur, ne pennis contemplationis per plateas supernae Sion volare possit. Sagittae diaboli sunt ira, invidia, luxuria et caetera quibus anima vulneratur». Voir aussi l'étude du thème à partir du Dit des sept Vices et des sept Vertus par Julia Bastin, "Trois "Dits" du XIII ${ }^{\mathrm{e}}$ siècle du manuscrit 9411-26 de la bibliothèque royale de Belgique», Revue belge de philologie et d'histoire, t. 20/3-4, 1941, p. 467-507, ici p. 490-492.

16. Méditation sur l'Ascension, $\S 3$. 
par degré, sans brûler les étapes, et commencer par se défaire par «humble penitence» des obstacles à l'amour de Dieu. Tel est le degré des «començans et imparfais ${ }^{17}$ - la purgatio - de l'échelle de vie contemplative que Gerson dresse dans sa Montagne de contemplation:

Qui veult commencier a savoir l'art de bien amer par la vie contemplative, doit tout premierement labourer a oster l'amour folle et contraire, que je puis appeler par mot general amour mondaine; car cest amour est comme gluz qui empesche les esles espirituelles de l'ame qu'elle ne puisse soi eslever en hault ${ }^{18}$.

La deuxième tentative s'ouvre donc par une petitio mieux adaptée au dénuement spirituel de l'impétrant, mais révélatrice aussi d'une détermination entière: «Si demande maintenent elles nouvelles et plumes pures et nectes et entieres a la samblance de la colombe». La modalité assertive qui apparaît alors à trois reprises ( «je ne veuil pas... je n'ay cure... je quiers ») et le parallélisme des propositions accentuent le contraste, dans le ton comme dans le rythme, avec le paragraphe précédent:

Je ne vueil pas les plumes d'autrusse, / par lesquelles je ne pourroye plus hault monter de terre: / ce sont les plumes d'ypocrisie.

Je n'ay cure des plumes du faucon ou de l'autour, / par lesquelles je ne pourroye repos trouver: / ce sont les plumes d'avarice et de rapacité;

ainçoys je quiers les plumes de la colombe, / les pennes de simplesse et de devote meditacion: / si voleray et me reposeray ${ }^{19}$.

L'effet de clôture est doublement travaillé: d'une part la symétrie des syntagmes est partiellement rompue dans la troisième phrase, qui énonce le véritable objet de la prière; de l'autre on observe le retour du thema en guise de clausule, mais un thema réduit à sa portion assertive, comme si, coupant court à l'effusion, il ne s'agissait plus que de mobiliser sa volonté et de formuler sa demande pour se voir aussitôt exaucé.

17. La Montagne de contemplation, $\$ 17$ (éd. P. Glorieux, op. cit., t. VII, $\mathrm{n}^{\circ} 297$, p. 27).

18. Ibid., $\S 11$, p. 22. Gerson revient plus loin sur l'image en accusant davantage le trait: celui qui s'imagine pouvoir faire l'économie de cette mortification s'expose à une cruelle désillusion, «car il voloient voler ainçois qu'il eussent elles, et batailler avec l'ennemi avant que ilz heussent seurmontés les aultres plus petis adversaires, le monde et la char» ( $\$ 28$, p. 37).

19. Méditation sur l'Ascension, § 4. 
L'illusion est de courte durée. La transition de la petitio à l'oratio, tout juste ménagée par une exclamation pathétique ( «las, je ne puis »), nous replonge aussitôt dans le lyrisme véhément de la méditation affective. On renoue alors, le temps d'une ample confessio, avec les rythmes ternaires, les balancements antithétiques, les phrases à concaténations et les emboîtements syntaxiques qui caractérisaient la facture du début:

Mon Dieu, mon maistre, mon pere de toute benignité, secourez moy ou descendez en bas a vostre povre fille consoler et relever, / ou m'octroiez plumes et elles moyennant lesquelles je puisse a vous vouler en hault, bien hault jusques au lieu secret de vostre demeure es sains cieulx lassus, ou doit estre la conversation de toutes ames devotes, la ou vous estes monté, vous qui estes leur amy loyal, le tout bel, le tout doulx, le tout bon, le tresglorieux espoux ${ }^{20}$.

Une fois de plus, le travail rythmique de la clausule relève de la prose d'art, en ce qu'elle prolonge et amplifie la cadence ternaire par le retardement du substantif qui prend dès lors une importance particulière, parce qu'il semble esquisser un thème, celui des noces mystiques. Emportée par sa ferveur et par le style qui l'informe, l'âme s'élève malgré tout.

D'où le mouvement de recul que marque l'interrogatio redoublée qui ouvre l'alinéa suivant: "Que di je, mescheante, que desiré je, que souhaidé je? Suis je digne de cest estat, de cest lieu, de cest honneur?». Un recul qui se révèle vite être une prise d'élan, tant ce qui suit déploie des ressources rhétoriques qui font paraître parcimonieux les développements précédents. La phrase s'étire en une succession de séquences ternaires au cœur de laquelle se niche un paradoxe, la conjonction de la crainte et de l'amour:

(...) sire et pere de toute consolacion, vostre piteuse misericorde me donne le congié d'ainsy parler et desirer; neccessité tresamere me contraint et vostre amour, l'amour de vostre presence, de vostre beaulté et de vostre compaignie me fait souspirer; a vous me fait gemir, plaindre et plourer, quant je me voy seulete desconfortee en ce present exil, en ceste vallee obscure et tenebreuse, en ce desert horrible plain de toute adversité, de toute misere et infelicité ${ }^{21}$.

La crainte et l'amour sont les deux forces qui renouvellent l'élan, tant affectif que syntaxique. Elles assument ainsi la fonction des 
deux ailes qui, selon Hugues de Saint-Victor, permettent l'élévation de l'âme dans la prière:

$\mathrm{Ne}$ ergo mens humana ad horandum pigra sit, hinc sua necessitate compellitur; ne autem trepida et diffidens oret, illinc pietate Dei provocatur. Istis duabus alis, miseria scilicet hominis et misericordia Conditoris, oratio sublevatur, quia dum mens alterna horum consideratione se ad devotionem incessanter excitat, quodam spiritualis desiderii impetu sursum levata volat ${ }^{22}$.

Au-delà de l'étape de purgation spirituelle, ces hauteurs nous laissent entrevoir le « lieu secret» et le silence qui caractérisent chez Gerson le deuxième degré de l'échelle de contemplation. En quoi on ne fait que dérouler le fil de sa méditation psalmique, qui poursuit au verset huit: Ecce elongavi fugiens et mansi in solitudine ${ }^{23}$. La solitude peut être toute intérieure ou physique, «par dedens ou par dehors $»^{24}$; c'est en tout cas une fuite - l'envol connote ici la promptitude - à laquelle doit se résoudre celui qui attend la venue de Dieu:

Et la vous atendré, qui me pouez sauver et delivrer de la paour et povreté de mon esperit et de la tempeste du monde, ou quel est iniquité et contradiction, labeur et injustice, usure et dolosité partout ${ }^{25}$.

La multiplication des binômes, parfois en plusieurs niveaux de détermination (voir les italiques) au sein d'une structure syntaxique à rallonge (deux relatives imbriquées, signalées en gras) donne une profondeur de champ à cette fuite, qui se réfugie dans l'espérance. On trouverait un contemptus mundi exprimé par la même image, mais sans les ornements des figures, dans le $D e$ fuga saeculi d'Ambroise: "Qui vult igitur manu Christi levari, ante ipse evolet, habeat pennas suas. Qui fugit saeculum, pennas

22. De virtute orandi, 1: «Ainsi, pour que l'esprit humain ne cède pas à la paresse dans la prière, il subit d'un côté la contrainte de la nécessité à laquelle il est soumis, et pour qu'il ne prie pas avec crainte et défiance, il est mû, d'autre part, par la bonté de Dieu. Avec ces deux ailes, la misère de l'homme et la miséricorde du Créateur, la prière prend son essor, car lorsque l'esprit ne cesse d'attiser sa dévotion en les considérant tour à tour l'une et l'autre, il s'envole vers les hauteurs, comme soulevé par le transport d'un désir spirituel» (L'œuvre de Hugues de SaintVictor 1, texte latin par Hugh B. Feiss et Patrice Sicard, traduction française par Dominique Poirel, Henri Rochais et P. Sicard, Turnhout, Brepols, 1997, «Sous la règle de saint Augustin», p. 128-129).

23. Ps. 54, 8: «J'ai fui au loin et j'ai demeuré dans la solitude».

24. Montagne de contemplation, § 23-24 (p. 32-33).

25. Méditation sur l'Ascension, $\S 6$. 
habeat $»^{26}$. Gerson en use aussi dans une lettre à Pierre d'Ailly, où il l'exhorte à «prendre les ailes de la colombe» et à trouver dans la solitude la «nourriture solide» de la dévotion:

Eh bien donc, illustre maître, fais ce que tu as entendu par le Prophète. Fais-toi des ailes semblables à celles de la colombe, les ailes de la méditation pieuse, afin de méditer comme la colombe, en pensant aux jours anciens et en gardant à l'esprit les années éternelles; c'est ainsi que tu t'en iras au loin, en faisant du Très-haut ton refuge, afin que le mal ne puisse s'approcher de toi. Délaisse par le mépris tout ce que tu peux librement délaisser parmi les choses terrestres. C'est ce que tu fais déjà ; mais il faut s'élever, et non demeurer où tu es. Si tu es arraché à la mamelle de la consolation terrestre, il te faut chercher une autre nourriture plus solide, celle de la dévotion intérieure ${ }^{27}$.

Mais l'image de la «tempeste du monde» sur laquelle Gerson s'attarde tant dans sa Méditation relève d'un registre plus ascétique: il ne s'agit pas d'adresser à son illustre mentor des mots de réconfort, mais d'éduquer l'âme novice et de la guider sur le chemin de la vie intérieure. Au terme de ce premier mouvement dominé par le lyrisme effusif de la méditation, l'apostrophe au corps vient donc mettre un point d'orgue à l'analyse introspective, en déployant l'intima locutio en un dialogue intérieur dramatique. Le corps entre en effet en scène sous les traits du partenaire rebelle, autre topos de la littérature ascétique:

Et tu, mon corps, qui souloyes estre mon amy par aucun temps sans moy empeschier a aler a Dieu, mais estoies d'un consentement avec moy en la voye et ou service de Dieu, tu mangoyes avec moy les doulces viandes des devos pleurs et des gemissemens a Dieu; la mort adviengne a ceulx qui te ont cecy fait, les faulx ennemis qui te font faire ceste guerre a moy, ce discort et ceste rebellion, en ce que tu

26. Ambroise, De fuga saeculi, V, 27-31 (PL 14, col. 584): «Qui veut être soulevé par la main du Christ, qu'il s'élève d'abord lui-même et se dote de ses propres ailes - qui fuit le siècle a des ailes». Dans la lignée d'Ambroise, Bernard de Clairvaux interprète les deux ailes comme étant le mépris du monde et l'affliction de la chair (Serm. in Epiph. 3, 6, 4, éd. Jean Leclercq et H. Rochais, Rome, Editiones Cistercienses, 1966, t. 4, p. 307).

27. Lettre du 16 oct. 1408 (ou 1411): «Age ergo, praeceptor inclyte, fac quod per prophetam audisti. Accipe pennas sicut columbae, pennae devotae meditationis ut mediteris sicut columba, cogitans dies antiquos et annos aeternos in mente habens [Ps. 76, 6]; sic enim elongabis, ponens Altissimum refugium tuum ut non possit accedere ad te malum [Ps. 90, 9-10]. Desere per contemptum quidquid in terrenis libere potes. Hoc jam facis; sed egrediendum est, non hic sistendum. Si ablactatus es ab ubere consolationis terrenae, quaerendus est cibus alius solidior, cibus internae devotionis » (P. Glorieux, op. cit., t. II, $\mathrm{n}^{\circ} 28$, p. 106). 
quiers maintenant tes plaisirs charnelz contre ma voulenté et en vagant partout es occupacions vaines ou nuisibles sans oïr mon contredit ${ }^{28}$ !

La vigueur de l'interpellation laissait présager une prise à partie violente; or c'est le ton de la nostalgie qui s'impose: un clivage temporel s'instaure entre un avant consensuel et harmonieux («avec moy») et un après où règne le «discort». Et si la phrase se relance en une imprécation («la mort adviengne...»), elle distingue le lieu physique du conflit et la cause première de la «division», les «faulx ennemis» qui détournent le corps de son obéissance à l'âme. Dans ce double conflit, à la mesure du désarroi intérieur, se joue aussi une leçon toute de modération sur la connaissance de soi : le corps n'est pas à mortifier par des ascèses excessives, car il est solidaire de l'âme, dans le mal (en tant que «charnalité corrompue») comme dans le bien (la paix de l'un est celle de l'autre: «nostre paix »). Il faut plutôt l'assujettir et le remettre à sa place, par la fermeté et la persévérance. Le style porte la marque de cette tension entre prière et injonction, de ce travail à la jointure de la chair et de l'âme: le je et le tu alternent de manière rapprochée, et les impératifs exhortatifs, d'abord mêlés à la modalité assertive, se font plus fréquents et rythmés (par l'anaphore) au fur et à mesure que renaît l'impatience de l'envol:

Racordons nous ensemble: trop mieulx nous estoit en nostre paix que en ceste division honteuse, que en ceste noyse et riote tresangoisseuse. Tu ne pues voler en hault, je le sçay bien, au moins jusques en la presence de mon amy, mais je te supply, ne me soyes point a destourbier! Sueffre que je recueille mes plumes de sainctes pensees et mes deux elles de desir et bon espoir; sueffre que je les nectoye et despesche ou que je en acquiere nouvelles: si m'en yray a mon amy; si voleray et me reposeray avec lui ${ }^{29}$.

L'ajout final est significatif: l'époux mystique s'est substitué au corps, une forme de compagnonnage à une autre («mon corps» / «mon ami», «avec moy»/ «avec lui»). Et l'écriture marque une halte avec le retour d'un thema-refrain glosé qui, par son amplification bipartite («si m'en yrai... si voleray...»), montre que l'âme a enfin pris son essor. 


\section{$2^{\mathrm{e}}$ DEGRÉ : L'ATTENTE ET LE DÉSIR \\ - FRAGMENTATION ET AMPLITUDE ORATOIRES}

La deuxième partie rompt doublement avec ce qui précède: d'abord en recadrant la méditation sur la fête de l'Ascension, jusqu'ici laissée à l'arrière-plan; en délaissant ensuite le style effusif et rythmé de l'introspection ascétique pour les contrastes et l'amplitude oratoire du sermon de tempore. Certes, le themarefrain, comme le Deo gratias qui conclut le texte dans le manuscrit de Saint-Victor, suggéraient d'emblée la trame d'un sermon. Il est donc légitime de s'interroger sur la nature même de l'œuvre et sur sa fonction, peut-être de l'ordre d'une ébauche homilétique. Quelle qu'en soit l'origine, une certaine hybridité demeure, et si l'on recense des procédés d'écriture typiques de l'ars praedicandi, on perçoit l'attraction d'un autre modèle, celui des Meditationes Vitae Christi $^{30}$. Ces changements ne sont pas gratuits. Ils participent aussi du mouvement ascensionnel en nous faisant franchir une nouvelle étape: nous voici désormais au deuxième degré de l'échelle de contemplation, dans ce «lieu secret», cet «entre deux états» fait de préparation et d'attente, où l'âme n'est «ne toute morte au monde, ne toute vive en Dieu $»^{31}$.

L'ancrage scripturaire nous fait lire le récit de l'Ascension à la lumière des Psaumes: le fil méditatif n'est donc pas rompu. Mais la méthode diffère: méditer l'Écriture, c'est avant tout «considérer» les faits en se faisant témoin des événements, aux côtés de la Vierge et des Apôtres; c'est se rendre présent à la scène en sondant dans son cœur les silences du texte:

Mon Dieu et mon espoux est monté es cieulx moult joyeusement et tresglorieusement, avecques lequel monsterent tous les anciens prophetes et patriarches et autres justes. Presens estoit, n'est point

30. Euvre longtemps attribuée à Bonaventure, due en réalité à un franciscain toscan de la fin du XIII ${ }^{\mathrm{e}}$ ou du début du XIV ${ }^{\mathrm{e}}$ siècle, les Meditationes Vitae Christi retracent la vie terrestre du Sauveur en intercalant au récit proprement dit des considérations pieuses qui sollicitent l'imagination et la compassion du lecteur. Avec la Vita Christi de Ludolphe de Saxe, les Meditationes furent les «catalyseurs de la religiosité du Moyen Age finissant» (G. Hasenohr, «La littérature religieuse», dans Grundriss der romanischen Literaturen des Mittelalters, t. VIII/1, Heidelberg, Carl Winter, 1988, p. 294). On s'appuiera ici sur l'édition de Mary Stallings-Taney: Jean de Caulibus (Pseudo-Bonaventure), Meditaciones vite Christi, Turnhout, Brepols, CCCM 153, 1997.

31. Montagne de contemplation, $\S 22$ (op. cit., p. 31) 
doubté, la compaignie des anges, et sembleroit que tous les prisonniers qui lors estoient en purgatoire furent delivrez, comme a la joye nouvelle de la feste bien appartenoit; sembleroit aussi que plusieurs y monterent a tout leurs corps gloriffiez, en especial ceulx qui ressusciterent aprés sa passion. Et vous, Vierge tresdigne, que dites vous a celle heure, quant vous le veïstes partir? Que dirent tous les appostres et les diciples aussi? Mains doulx souspirs y ot, c'est bien a croire, mains regrez, mains souhaiz semblables aux miens mais trop plus grans, pour s'en vouler avec lui lassus, mesmement corporellement se son plaisir y eust esté ${ }^{32}$.

Le texte adopte d'emblée une nouvelle facture, où prédominent la modalité assertive et les temps du récit. Le style linéaire énumère les actants dans un ordre hiérarchique: 1) le Christ d'abord, les prophètes et patriarches, suivis des justes anonymes; 2) les anges; 3) les âmes délivrées du purgatoire; 4) les ressuscités après la Passion. On reconnaît la prudence de Gerson aux marques de modalisation dont il parsème sa description. La première phrase, qui n'en comporte aucune, se fonde sur la première Épître de Pierre ${ }^{33}$, d'où procède la doctrine de la descente du Christ aux «limbes des Pères», abondamment alimentée par la littérature apocryphe ${ }^{34}$. L'incise «n'est point doubté» introduit une première distanciation qui s'appuie sur l'autorité de la Glose, reprise largement dans la Légende dorée de Jacques de Voragine ${ }^{35}$. Enfin, l'incertitude est assumée par le verbe «sembler» (au conditionnel pour en renforcer la virtualité), dont la reprise assure la relance au sein même de la concaténation. L'argument de convenance («comme a la joye de la feste bien appartenoit») est situé à sa place, avant l'emprunt à

32. Méditation sur l'Ascension, $\S 8$.

33. 1 P 3, 19: «c'est alors qu'il est allé prêcher même aux esprits en prison» (trad. TOB). La descente du Christ aux Enfers est évoquée ailleurs dans le Nouveau Testament (Act. 2, 31; Rom. 10, 7 ; Éph. 4, 8-10), mais le verset en question est le seul à suggérer une intervention du Christ pour en délivrer les âmes.

34. En particulier le Pseudo-Augustin, Sermones suppositi de tempore, Sermo 160, 2 (PL 39, col. 2060) et L'Évangile de Nicodème, XVII et suiv. (éd. et trad. Rémi Gounelle et Zbigniew Izydorczyk, Turnhout, Brepols, 1997).

35. Jacques de Voragine, Légende dorée, 67, 4: «Sur le quatrième point en compagnie de qui est monté au ciel -, il faut noter ceci : il y est monté avec un grand butin d'hommes et une grande multitude d'anges » (éd. et trad. sous la dir. d'Alain Boureau, Paris, Gallimard, «La Pléiade», 2004, p. 384), et la Glose marginale à Is. 63, 1 (Biblia latina cum Glossa ordinaria. Anastatical Reproduction of the First Printed Edition, Strasbourg, 1480, Turnhout, Brepols, 1992, t. III, p. 91a). 
L'Évangile de Nicodème ${ }^{36}$, apocryphe dont la contribution est minorée comme il se doit.

Mais la prudence n'exclut pas un appel raisonné à l'imagination, comme le montre l'apostrophe à la Vierge. Souvent interpellée dans les sermons de Gerson $^{37}$, Marie constitue le point focal de la méditation affective: c'est de son émotion que se nourrit l'imitation. Mais sur ce point encore, Gerson maintient une certaine distance, signalant par une incise modalisatrice («c'est bien a croire») la part de projection rétrospective que comporte nécessairement toute méditation («mains regrez, mains souhaiz semblables aux miens mais trop plus grans»). L'écart est manifeste avec le chapitre 105 des Meditationes Vitae Christi de Jean de Caulibus:

Que dirais-je au sujet de sa mère qui s'était assise à table près de lui, et qui l'aimait de tout son cœur et plus que tout au monde? Ne crois-tu pas qu'en entendant les paroles de son fils sur le point de partir, mue par l'amour maternel et dans un élan de tendresse, elle ait incliné la tête et l'ait laissée reposer sur sa poitrine? [...] En soupirant, au milieu de ses larmes, elle l'implorait ainsi: "Mon fils, si tu veux t'en aller, emmène-moi avec toi". Et le Seigneur lui disait pour la consoler: "Je t'en prie, mère bien aimée, ne t'afflige pas de mon départ, car je m'en vais auprès du Père" 38 .

L'émotion de Marie ne donne pas lieu chez Gerson à un dialogue entre le Christ et sa mère. Mais elle suffit à donner sens à la métaphore matrice: la douleur de la séparation est surmontée par l'envol de l'âme dotée des «pennes treslegieres de contemplacion». Là s'arrête pour Gerson la légitimité de l'imagination.

36. Évangile de Nicodème (éd. cit., p. 180): «Il faut avec raison s'étonner encore plus de ce que non seulement il est ressuscité des morts, mais qu'il a ramené vivants de nombreux autres morts de leurs tombeaux à la vie, et que beaucoup les ont vus à Jérusalem». La suite rapporte le témoignage de Leucius et Carinus, les deux fils ressuscités du grand-prêtre Syméon.

37. L'exemple le plus frappant est celui du sermon sur la Passion Ad Deum vadit (éd. P. Glorieux, t. VII*, n 341, p. 449-519).

38. Jean de Caulibus, Meditaciones vite Christi, ch. 105 (éd. cit., p. 337, 1. 44-52): "Quid autem dicam de matre iuxta eum prandente, que super omnes sic intense amabat? An non credis quod ad hec verba discessus filii, materne dileccionis tacta et commota dulcedine reclinaret caput suum et recumberet super pectus eius ? [...] Suspirans cum lacrimis rogabat eum, dicens: "Fili mi, si discedere vis, ducas me tecum". Dominus autem consolans eam, dicebat: "Rogo mater carissima, ne feras moleste discessum meum, quia vado ad Patrem" ». 
Même sobriété - accommodée d'un certain lyrisme - dans l'évocation du chant des anges accompagnant le Christ dans son élévation:

Les sains anges et archanges quelle feste firent ilz, quelle melodie chanterent en regardant comment tous ceulx que Jhesucrist menoit de sa livree, ceulx de l'umain lignage, n'est point a recyter ne souffisamment penser $^{39}$.

Une fois de plus, le contraste est net avec le traitement des Meditationes Vitae Christi, qui font longuement entendre les louanges angéliques, sous forme d'un centon scripturaire:

en s'inclinant avec toute la déférence dont ils étaient capables, ils l'escortaient en chantant hymnes et cantiques ineffables. Qui pourrait en effet reproduire les chants et les jubilations qu'ils proféraient? Venant à leur rencontre, les princes chantaient et disaient: "Alléluia, alléluia, alléluia! Béni soit celui qui marche sur les ailes des vents. Alléluia, alléluia! Tu es digne, Seigneur, d'être loué et honoré de tous. Alléluia! Car tu as triomphé dans la gloire. Alléluia! Les cieux confessent tes merveilles et ta puissance, Seigneur" ${ }^{\prime 2}$.

Si l'écart est revendiqué par Gerson («n'est point a recyter ne souffisamment penser») par souci de fidélité au texte canonique, c'est ici une habile prétérition qui met en valeur un détail important de la scène: le ciel «sans nuee» qui s'ouvre devant l'âme est celui du troisième degré de l'échelle de contemplation - «forte persévérance $»^{41}$ - étape encore à venir («bien eureuse sera l'ame qui ainsi les eschevera»), mais promesse qui tranche sur l'accablement de la première partie. L'exclamatio finale traduit la vigueur de l'élan retrouvé. L'intériorisation de l'Ascension a pris le pas sur les «ennemis d'umain lignage».

39. Méditation sur l'Ascension, $\$ 8$.

40. Meditaciones vite Christi (éd. cit., p. 339, 1. 106-113): «inclinantes se cum omni qua poterant reverencia deducebant eum cum hymnis et canticis ineffabilibus. Quis enim posset cantus et iubilos qui ab ipsis fiebant explicare? Adveniendo ergo principes coniunctu psallebant et dicebant: "Alleluia, alleluia, alleluia. Benedictus es qui ambulas super pennas uentorum [Ps. 103, 3]. Alleluia, alleluia. Dignus es Domine omni laude et honore [Apoc. 5, 11]. Alleluia. Quia uictoriam gloriose fecisti. Alleluia. Confitebuntur celi mirabilia tua Domine et virtutem tuam" [Ps. 88, 6]».

41. Montagne de contemplation, § 33: «En cest estat art l'ame devote doucement et souefment et purement dou feu esprituel, comme un carbon cler et embrasé et sans fumee ou sans bruire [...]. On puet aussi comparer le premier estat au jour tel comme il est au matin quant la clarté est enveloppee de tenebres et ne commence que apparoir moult obscurement; le second estat est samblable au jour de tierce; li tiers au jour de midy» (éd. cit., p. 41). 
L'exemplum qui, sans transition, succède à cette relecture de l'ascension du Christ, fait une trouée dans la trame discursive. Par sa nature, d'abord, il fournit un autre point de référence que celui qui jusqu'alors était en place: au temps biblique succède un «jadis» qu'on ne peut situer précisément dans la mémoire collective («comme on recite»), mais qui s'inscrit dans le temps de l'Église. Par son mode d'écriture, ensuite: une narration brève, sans fioritures, dont l'efficacité exemplaire tient à l'interprétation qu'en fait Gerson dans le corps même du récit. La mort d'amour du «pèlerin de l'Ascension» devient le témoignage d'un envol assez puissant par sa ferveur pour détacher l'âme de son enveloppe matérielle. En confrontant cette version avec celle qu'en donne le $\mathrm{Ci}$ nous $\mathrm{dit}^{42}$, on repère aisément les inflexions lexicales par lesquelles Gerson fait servir l'exemple à sa démonstration:

Jadis, comme on recite, une devote personne, aprés ce qu'elle ot visité les sains lieux ou nostre sauveur Jhesucrist avoit esté corporellement, vint a la parfin ou mont d'Olivet. Illec adoura le lieu ou les benois piés de Jhesus avoient esté, et apparoient les traces. «Ou vous querray je plus, Sire? dit ceste devote personne; je ne sçay plus ou aler ce je ne vole a vous.» Lors elle esmut tant fort ses elles de saint desir que l'ame party du corps et s'en vola. // O qui me pourra donner telles puissans eles? Si voleray et me reposeray. ${ }^{43}$
$\mathrm{Ci}$ nous dit conment un sainz chevaliers visita de son pouair touz les lieus ou li debonnaires Jesucriz avoit esté viz et mors. Et par devotion se coucha seur le mont d'Olivet, en grans larmes disant a Nostre Segneur: Sire, je vous ai quis de mon pouair par touz les lieuz ou vous fustez viz et mors. Si ne vous soi plus ou querir. Vous vous en alastes d'ici; si allasse voulentiers a vous se je peusse. Et en ce disant avoit si grant ardeur en son cuer de devotion qu'en celle heure trespassa sainctement. // Si li empli Nostre Sire son bon desirier et le mist en possession dou pardurable heritage dou ciel quar tant avoit desirré.

Le brusque surgissement du thema-refrain en guise de conclusion, pour remplacer le motif de la récompense céleste du Ci nous dit, achève de renforcer la cohérence du discours. L'ajustement est net et précis: ce qui importe ici, c'est la puissance des ailes, et non plus leur nature ou l'objet qui les meut. L'impact est d'autant plus

42. Ci nous dit. Recueil d'exemples moraux, éd. Gérard Blangez, Paris, Société des Anciens Textes Français, 1986, t. II, ch. 472, p. 52.

43. Méditation sur l'Ascension, $\$ 9$. 
fort, et le retour sur soi plus violent, que la brusque réapparition du discours par le biais de l'interrogatio psalmique s'autorise d'une proximité référentielle absente du développement précédent: derrière l'anonymat de cette «devote personne», on discerne la figure du pèlerin, familière à chacun. Dans la version qu'il donne de l'histoire, Thomas de Cantimpré va même jusqu'à l'identifier par son origine et situer précisément cette «mort de compassion»: il s'agirait d'un vir fidelis et bonus natif de Dinant qui aurait «traversé la mer» en $1216^{44}$. Gerson se contente de reproduire la trame de l'épisode, laissant au méditant le soin d'en restituer les détails.

Non content d'illustrer ainsi son propos, Gerson utilise aussi l'exemplum pour relancer le cours de la méditation par un nouveau renversement dramatique:

Mais helas puis que je, povre ame orphenine et vesve, n'ay point ceste grace, ceste force et ce congié de m'en voler hors de mon corps avec mon doulx espoux, le vray amy de mon cuer, je feray au moins a l'exemple de Nostre Dame et des appostres ${ }^{45}$.

À ce qui reste un cas de ferveur admirable pour la grâce singulière dont elle témoigne, mais qui s'avère dès lors impossible à imiter, il faut opposer un modèle plus accessible. La différence est mince en apparence avec une rechute dans le désarroi initial: la reprise des termes «orphenine et vesve» nous y ramènerait-elle? Mais l'ascension se poursuit, et la démarche d'imitatio inaugurée entre temps peut se prévaloir désormais d'une intériorisation du modèle: c'est la Vierge et les apôtres - et non le Christ, hors de portée - qu'il s'agit de prendre pour «exemple».

S'ouvre alors une ample continuatio ${ }^{46}$ qui, sur le plan de l'écriture, constitue le sommet de l'œuvre et alimente, pour ainsi dire, l'imitation par le style. Fondée toute entière sur le procédé de la moralisation allégorique, la période oratoire fait basculer le texte par son agencement, d'un «avant» marqué par la tension vers les hauteurs inaccessibles où disparaît le Christ, à un «après» dévolu

44. Thomas de Cantimpré, Bonum universale de apibus, I, 15, 5: «De viro, qui Christo compatiendo obiit» (éd. Georges Colvenere, Douai, 1627, p. 102-103).

45. Méditation sur l'Ascension, $§ 10$.

46. Sur ce terme latin désignant la période considérée comme une succession continue d'éléments formant une unité sémantique et rythmique, voir en particulier la Rhétorique à Herennius, IV, 27 (éd. Guy Achard, Paris, Les Belles Lettres, 2003, p. 161-162). 
à l'approfondissement intérieur, seule voie possible pour s'unir à l'époux mystique. Le schéma suivant permet de faire apparaître la structure anaphorique de la phrase et sa cadence équilibrée (six cola dans les deux séquences, ménageant chaque fois un effet de descrescendo/crescendo des commata $^{47}$ ):

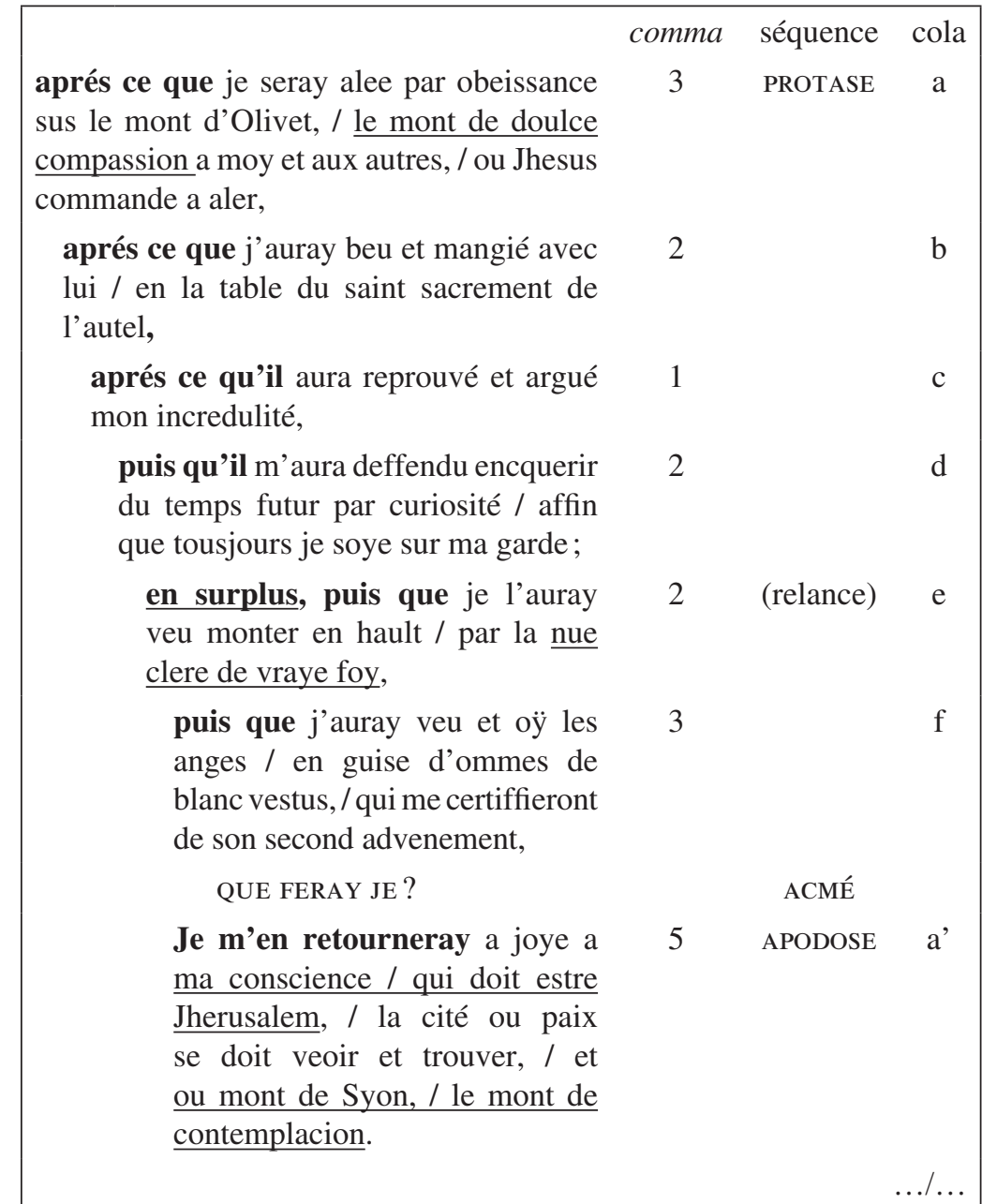

47. On emploie ces deux termes, hérités de la rhétorique antique, pour distinguer deux niveaux de segmentation de la phrase périodique: le côlon ou membre constitue une sous-partie de la période, de longueur variable; il se fragmente à son tour en un certain nombre de commata ou incises (indiqués par des traits obliques). 

comma séquence La m'enfermeray je ou grant 2 senacle, / ou senacle de charité large et espandue;

je fermeray les portes de mes cinq sens pour la paour dez juifs, / c'est assavoir de tous mes adversaires.

Illec en seant et en priant je actendray / jusques a tant que je soye revestue et visitee de la vertus d'en hault du Saint Esprit ;

j'actendray qu'il tonne si hault / que je m'esmeuve toute par saincte paour / et que ma sorde oreille soit ouverte a ce grant son;

je actendray qu'il envoye feu si cler et si vif et si ardant / que je soye toute enluminee, animee et embrasee, / affermee contre tous maulx [et aux louanges divines tresbien enlangagee.]

2

2

3

$e^{\prime}$

4

L'art est bien celui du sermon ${ }^{48}$ : outre la facture rhétorique de la période elle-même, on note que le séquençage s'appuie sur le procédé de l'emblématisme allégorique ${ }^{49}$, déjà introduit dans la première partie par l'intermédiaire du bestiaire. Mais la visée va au-delà du discours d'édification morale dont Gerson est coutumier. Elle est d'abord exégétique, en ce que l'on a affaire à une longue phrase à fonction charnière, dont la dynamique traduit le mouvement qui mène de l'Ascension à la Pentecôte. En effet, l'Ascension, qui donne lieu à un récit répété à la jonction entre les deux volets de l'œuvre lucanienne (Lc 24, 50-53 et Act. 1, 6-11), signifie à la fois «l'apogée de la seigneurie de Jésus et l'instauration de son absence», la conclusion de son activité et une «ouverture au temps du témoignage $»^{50}$. On regarde à la fois vers le temps de la Passion

48. Sur le style oratoire de Gerson, voir l'étude déjà ancienne de Louis Mourin, Jean Gerson prédicateur français, ch. 5: «Le style», Bruges, De Tempel, 1952, p. 427-496.

49. Armand Strubel, La Rose, Renart et le Graal. La littérature allégorique en France au XIII siècle, Genève-Paris, Slatkine, 1989, p. 55-58.

50. Daniel Marguerat, Introduction au Nouveau Testament. Son histoire, son 
et vers celui de la Pentecôte, et c'est cette double lecture qui fonde justement l'itinéraire de l'âme: le «feu si cler et si vif et si ardant» dont elle appelle la venue et auquel elle se dispose est celui-là même qui brûle au sommet de la «montagne de contemplation».

\section{$3^{\mathrm{e}}$ DEGRÉ : APPROFONDISSEMENT ET RETOURNEMENT DU THÈME - LE TRAITÉ DE THÉOLOGIE MYSTIQUE}

Les trois derniers paragraphes tranchent sur l'ample développement oratoire de la section précédente. Ils se distinguent aussi par la disparition du je et des marques de subjectivité dans le discours. Rompant avec un style affectif marqué par une certaine emphase, qui multipliait les gradations et les cadences ternaires, les tours exclamatifs et interrogatifs, le discours se fait plus linéaire et démonstratif; les phrases simples s'enchaînent sans subordinations complexes, à grand renfort de connecteurs argumentatifs. Il ne s'agit plus de peindre les tâtonnements d'une âme qui se cherche, mais de donner à voir l'aboutissement d'un chemin. Les images se font plus rares et assument une fonction principalement cognitive: la figure de l'âme-oiseau laisse place à un lexique abstrait connotant le mouvement d'élévation. À l'exception du dernier paragraphe, qui voit la résurgence de l'emblématisme allégorique (on verra pourquoi), le ton et l'écriture sont bien ceux du traité didactique. La matière ne nous surprendra pas, s'agissant de Gerson: on se situe dans le cadre de son traité de théologie mystique.

Le premier alinéa énonce le paradoxe de l'approfondissement apophatique à travers le motif de «l'abîme sans fond». L'envol de l'âme n'est plus une figure; il est l'expression imagée d'un mouvement noétique qui, une fois engagé, trouve sa vérité dans son inaccomplissement:

Quant la saincte ame monte en la hautesse de son cuer par contemplacion, Dieu l'eslieve en hault, selon le dit du Prophete; car tant plus semble Dieu hault et incomprenable a icelle comme elle s'efforce plus de l'aprouchier. Mais ne s'en plaint point l'ame, ainçoys s'en tourne plus a l'onnorer, a le doubter et a mercier. Si est Dieu, qui ne puet estre comprins, tant plus cognu comme on espreuve que il ne peust estre comprins ou tout cogneu, ainsi comme ung abisme sans

écriture, sa théologie, ch. 6: «Les Actes des Apôtres", Genève, Labor et Fides, $2008^{4}$, p. 128. 
fons donne congnoissance merveilleuse aux regardans, en ce que plus $\mathrm{y}$ regarde on et moins on puet son fons veoir ${ }^{51}$.

Appréhendée comme «parfaite inconnaissance», la «contemplation » nie son propre objet pour le saisir dans son altérité. Le plus haut point de la connaissance de Dieu est de comprendre que Dieu déborde à l'infini tout ce que l'on peut en concevoir et en dire : c'est le thème de la «docte ignorance» qui, dès lors qu'elle se connaît, est une forme de savoir. On le trouve exprimé chez Augustin (Deus qui scitur melius nesciendo ${ }^{52}$ ), mais c'est le Pseudo-Denys qui lui donne sa portée spéculative la plus grande:

S'il advient que, voyant Dieu, on comprenne ce qu'on voit, c'est qu'on n'a pas vu Dieu lui-même, mais quelqu'une de ces choses connaissables qui lui doivent l'être. Car en soi il dépasse toute intelligence et toute essence; il n'existe, de façon suressentielle, et n'est connu, au-delà de toute intellection, qu'en tant qu'il est totalement inconnu et qu'il n'existe point ${ }^{53}$.

L'image des ténèbres lumineuses ou celle de la «souveraine silence», lieu du parfait unisson ${ }^{54}$, sont récurrentes chez Gerson pour rendre compte du paradoxe. Mais il choisit ici une autre voie, donnant à entendre par le style le processus même de la voie dite «négative». La pensée est prise dans un jeu de balancements entre les deux membres des comparatives qui, au premier abord, semblent s'annuler l'un l'autre («tant plus... incomprenable... comme... plus de l'aprouchier», «tant plus cognu comme... ne peust estre comprins ou... cogneu», «plus y regarde on... moins on puet... veoir»). La perplexité du copiste du manuscrit BnF, fr. 990 suffit à témoigner du caractère retors de cette mimesis stylistique, qui joue aussi du procédé de la réduplication ( «comprins» et «cognu» répétés et niés dans la même phrase): il laisse à cet endroit un blanc d'une ligne et demi, ne retrouvant pied, si l'on ose dire, qu'avec l'image de l'abîme.

51. Méditation sur l'Ascension, $\S 11$.

52. Augustin, De Ordine, II, XVI, 44 (Bibliothèque Augustinienne 4/IV, éd. René Jolivet, Paris, Desclée de Brouwer et Cie, 1948, p. 438): «Dieu qui est bien mieux connu en ne l'étant pas » et la note complémentaire XI (p. 465). Voir aussi Thierry-Dominique Humbrecht, Théologie négative et noms divins chez saint Thomas d'Aquin, Paris, Vrin, p. 68 («Le cas d'Augustin»).

53. Denys, Lettre I «À Gaios» (1065 A), dans CEuvres complètes du PseudoDenys l'Aréopagite, trad. Maurice de Gandillac, Paris, Aubier, 1943, p. 327.

54. Canticordum au pelerin, XLIX-5, dans Isabelle Fabre, La doctrine du chant du cœur de Jean Gerson, Genève, Droz, 2005, p. 516. 
Le processus d'élévation ne s'approfondit pas seulement sur le plan de la noétique; il s'accomplit également à l'échelle de l'universel, en une reductio qui témoigne de la vocation première de l'âme à regagner sa source divine :

En ceste elevacion de terre en hault par contemplacion, l'ame trait tout a soy mesmes, car n'est chose lors que l'ame voye ou oye ou sente ou gouste ou taste ou flaire ou pense que elle ne torne tout a son prouffit, a soy lever en hault pour Dieu mieulx assavourer, sentir et desirer. Par le contraire est de l'ame qui verse a terre scienment, car de tout fait son dommage; tout s'apesantist sur elle pour elle confondre et empeschier a relever ${ }^{55}$.

La notion de reductio ou de «retour», telle que Bonaventure l'élabore dans son Itinerarium $^{56}$, est de nature ontologique: elle concerne le statut particulier de la créature raisonnable, seule capable d'accomplir, en tant que microcosme, l'unification en Dieu de toute la création. Gerson revient longuement sur cette opération dans son commentaire sur la Hiérarchie céleste:

L'homme ramène toutes les choses corporelles à la simplicité divine par la connaissance et l'amour, d'abord quand il reçoit par ses sens tout le monde sensible, puis il le simplifie selon les modalités que l'on a vues. C'est pourquoi l'on dit que tout a été fait pour l'homme, que l'homme est un petit monde, et qu'il est le nœud entre deux mondes, le corporel et le spirituel ${ }^{57}$.

Mais la Méditation sur l'Ascension n'est pas un traité de théologie mystique; si elle en reprend certains termes clés, c'est pour leur faire servir un tout autre propos, qui est de l'ordre de l'édification. La «contemplation» gersonienne peut recouvrir des

55. Méditation sur l'Ascension, $§ 12$.

56. Étienne Gilson, La philosophie de saint Bonaventure, Paris, Vrin, 1953², p. $353-378$

57. Notulae super quaedam verba Dionysii de Caelesti Hierarchia, dans André Combes, Jean Gerson commentateur dionysien. Pour l'histoire des courants doctrinaux à l'Université de Paris à la fin du XIV siècle, Paris, Vrin, 1973, p. 329-330: «Homo reducit omnia corporalia per cognitionem et amorem in divinam simplicitatem, dum primo recipit per sensus totum mundum sensibilem, postmodum simplificat modis tactis. Hinc dicuntur omnia facta propter hominem. Hinc dicitur esse minor mundus. Hinc est dictus nexus duorum mundorum, spiritalis et corporalis ». Voir aussi le sermon pour le Jeudi Saint A Deo exivit (éd. P. Glorieux, t. V, n 208 , p. 14): «Anima humana praecipue illa est inter omnes rationales spiritus quae non solum regredi ad Deum, sed omnia alia in eum referre debet» ( «C'est surtout l'âme humaine qui, entre tous les esprits rationnels, non seulement retourne à Dieu, mais doit y faire retourner toutes les autres choses »). 
réalités très diverses; elle opère avant tout sur le plan affectif : l'âme fait «son prouffit» du sensible en ce qu'elle aime Dieu davantage, mais elle ne le «réduit» pas à proprement parler par une opération hiérarchique $^{58}$. La polysyndète et l'expansion accumulative qui l'expriment («voye ou oye ou sente ou gouste ou taste ou flaire ou pense») font parcourir le spectre sensoriel en lui insufflant un élan que la clausule ternaire («pour Dieu mieulx assavourer, sentir et desirer») équilibre harmonieusement. La description opposée de l'âme «qui verse a terre sciemment» montre bien que l'enjeu n'est pas d'ordre ontologique, mais bel et bien ascétique: le lexique de l'accablement moral qui réinvestit le texte à ce moment-là («apesantist», «confondre et empeschier a relever»-l'image de la glu n'est pas loin) est là pour rappeler que c'est de la métaphore psalmique que procède la cohérence du propos. La concaténation ( «de tout fais son dommage, tout s'apesantist sur elle pour elle confondre... ») achève de mimer le lien qui, même à ce stade de la progression, demeure à l'arrière-plan de la démarche.

Mais on n'a pas encore atteint le terme et le motif de l'homme microcosme (que l'écriture traduit à nouveau par le biais de la polysyndète) sert d'embrayeur pour une ultime «envolée» :

Creature humaine est un petit monde qui est raempliz de tant de gens comme dedans elle sont cogitacions et affeccions et pensees et ymaginacions et voulentés diverses. Jhesucrist doncques, quant monte en hault dedans l'ame jusques au souverain ciel de l'esperit, qui est la plus haulte partie de raison car elle touche a Dieu sans moyen, l'ame est envoyee preschier et denoncier la loy de l'Evangille a toutes gens ${ }^{59}$.

Une envolée qui s'appuie cette fois sur un retournement total de l'image. À la question du qui, quo et ubi de la contemplation, on peut donner maintenant une autre réponse: ce n'est pas l'âme qui monte vers le ciel pour y rejoindre le Christ; c'est le Christ qui monte en l'âme «jusques au souverain ciel de l'esperit » afin d'y faire éclore de nouveaux charismes. L'expression a de quoi surprendre; elle nous renvoie à un modèle noétique, celui du Benjamin major de Richard de Saint-Victor ${ }^{60}$, exploité par Gerson dans sa Théologie mystique,

58. La hiérarchisation des facultés internes de l'âme est présentée dans les opuscules et traités mystiques de Bonaventure (Itinerarium, De triplici via, Breviloquium, etc.), par analogie avec la hiérarchie angélique.

59. Méditation sur l'Ascension, $\S 13$.

60. Benjamin major, I, 9 (PL 196, col. 74C). À noter que Richard compare les six genres de contemplation qu'il définit dans son traité à trois paires d'ailes 
où elle constitue le degré le plus élevé dans l'ordre des puissances cognitives de l'âme, sous le nom d' «intelligence simple»:

L'intelligence simple est une puissance cognitive de l'âme, recevant immédiatement de Dieu une certaine lumière naturelle, dans laquelle et par laquelle les premiers principes sont connus comme étant vrais et très certains, sitôt que les termes en ont été appréhendés ${ }^{61}$.

Ce celum supremum nous situerait donc dans le cadre d'un autre type de contemplation, mettant en jeu les puissances cognitives plutôt qu'affectives. Mais la distinction, typiquement gersonienne, ne renvoie pas à une diversité réelle; c'est en réalité la même puissance qui se dédouble à son sommet: à la contemplation intellectuelle, réalisée au niveau de l'intelligence simple, correspond l'amour extatique, par l'actualisation de la plus haute puissance affective de l'âme qu'est la syndérèse ${ }^{62}$.

Cependant le terme est absent du texte, preuve s'il en était besoin que l'enjeu concerne moins la connaissance théorique que la reformatio du sujet. Renouant avec la fête qui sert de «matière» à sa méditation, Gerson moralise l'envoi des Apôtres au moment du départ du Christ: appliquée au microcosme, la mission apostolique concerne autant les «cogitacions» et pensées que les affections et désirs, autant de «gens» qui peuplent le «petit monde» qu'est l'homme et qui doivent être «baptisés». La discipline intérieure suppose pour Gerson une méthode rationnelle; il est juste qu'elle s'exerce dans «la plus haute partie de raison», mais une raison

qui élèvent l'homme jusqu'aux mystères divins: «Oportet absque dubio omnes illas superius designatas sex contemplationum alas habere qui cupit et ambit usque ad tertii coeli secreta Divinitatisque arcana volare.» (I, 10, col. 76D). «Il est absolument nécessaire qu'il se munisse des six ailes de la contemplation dont nous avons parlé plus haut, celui qui désire et souhaite voler jusqu' aux secrets du troisième ciel et atteindre les arcanes de la divinité ».

61. De mystica theologia speculativa II, 10: «Intelligentia simplex est vis anime cognitiva suscipiens immediate a Deo naturalem quamdam lucem, in qua et per quam principia prima cognoscuntur esse vera et certissima, terminis apprehensis » (trad. Marc Vial dans Jean Gerson, Sur la théologie mystique, Paris, Vrin, 2008, p. 77).

62. Ibid., consid. 27, p. 86-87: «Synderesis est vis anime appetitiva immediate a Deo suscipiens naturalem quamdam inclinationem ad bonum, per quam trahitur insequi motionem boni ex apprehensione simplicis intelligentie sibi presentati» (trad. M. Vial: «La syndérèse est la puissance appétitive de l'âme recevant immédiatement de Dieu une certaine inclination naturelle au bien, par laquelle elle est poussée à suivre la motion du bien sur la base de ce que l'intelligence simple lui a présenté»). 
envisagée indissolublement comme puissance cognitive et affective, éclairée de surcroît par l'Esprit de la Pentecôte. D'où le recours in fine à l'emblématisme allégorique, qui énumère avec toute la netteté requise l'effet des différents charismes par lesquels l'âme peut désormais se «régler»:

[...] l'ame est envoyee preschier et denoncier la loy de l'Evangille a toutes gens pour les bien rigler et ordonner et pour les baptisier et laver en l'eaue de sainctes lermes, ou non du Pere et du Filz et du Sainct Esperit. Reçoyt en oultre l'ame telle puissance et telz signes, car elle chace hors les ennemis par les exhorcismes d'oroisons; elle parle langage nouvel, langage du ciel; elle oste les serpens des venimeuses pensees; elle guarist toute enfermeté dedans soy par les mains de bonne operacion $[\ldots]^{63}$.

Les connecteurs logiques et les verbes au présent confortent la valeur de vérité d'un énoncé qui s'actualise dans le temps même de la méditation. Enfin le dernier alinéa, noté comme tel dans le manuscrit, est le point de départ d'une gradation dans laquelle on peut voir la trace d'un envol désormais accompli : les cinq «puissances» exercées par l'âme s'étagent en un crescendo syntaxique aux effets de symétrie savamment perturbés (voir la rupture introduite dans le parallélisme par le deuxième colon «elle parle langage nouvel, langage du ciel», par épanorthose). L'ensemble culmine avec l'expansion de la séquence hypothétique, à la cadence ternaire et au dernier comma sensiblement plus long que les deux précédents:

$[\ldots]$ et se elle boit aucun venin mortel par mauvaise suggescion ou temptacion, / rien ne lui nuist a mort, / car ce venin ne va point jusques au vif du cuer par mauvais consentement et deliberacion parfaicte ${ }^{64}$.

Cette «immunité» spirituelle atteste le chemin accompli depuis le premier degré de l'échelle de contemplation. Est-ce pour autant le fin mot de l'affaire? Le texte ne se clôt pas sur un retour ultime du thema-refrain, et malgré l'ample mouvement du dernier paragraphe, la conclusion reste abrupte, si l'on peut même parler de conclusion. Resterait-on en «suspens», comme pétrifié dans l'envol? Ou l'impasse apparente signale-t-elle une volonté délibérée de taire ce qui se joue au-delà? On songe au décrochage des notules sur la «haute gamme» du Canticordum au pelerin: parole plus rare, style coupé, assertif, jouant des paradoxes mêmes de l'inconnaissable -

63. Méditation sur l'Ascension, $\S 13$.

64. Ibid. 
autant d'éléments qui manifestent ce «mouvement anagogique» qu'est pour Gerson la théologie mystique ${ }^{65}$.

Mais si le chancelier utilise le vocabulaire et les concepts de la théologie mystique, c'est, comme on l'a dit, avec une visée d'édification morale qui relègue au second plan les préoccupations «mystiques», tout en conférant à la dynamique de la Méditation une réelle unité. C'est aussi un apport singulier, qui permet de cerner de plus près la date de composition de notre opuscule. En prenant comme terminus ante quem le premier état du traité De mystica theologia (1408) et pour terminus ad quem l'Epistola 55 (1425), qui marque un «bouleversement» dans la noétique gersonienne ${ }^{66}$, on pourrait rapprocher notre opuscule du sermon pour la Pentecôte Spiritus Domini (1416) ${ }^{67}$, qui réserve une place décisive à l'action de la grâce, et plus particulièrement du SaintEsprit, dans le processus d'acquisition de la théologie mystique. Mode de connaissance le plus élevé auquel l'homme ait accès en cette vie, la «contemplation» implique l'actualisation du versant cognitif de la puissance la plus haute de l'âme. Mais l'extase mystique proprement dite suppose de s'ouvrir à l'amour divin. Le texte ne dit pas comment, mais il suggère en fin de compte un envol qui dépasse la sphère des intelligibles: munie des ailes nouvelles que lui confère l'Esprit, l'âme peut s'élever au-dessus d'elle-même et parvenir en Dieu, le lieu de son repos.

Sous les dehors d'un opuscule conventionnel, exploitant la topique bien connue de l'âme-oiseau, la Méditation sur l'Ascension de Gerson laisse apparaître un discours hybride, convoquant plusieurs types d'écriture, mais doté d'une cohérence profonde. Nourrie du style de la méditation ascétique, à la fois effusif et heurté, la prose rythmée prend en charge les aléas de la progression du méditant, du motif initial des ailes engluées à la montée vers le troisième ciel de l'âme rationnelle, en passant par l'étape de solitude

65. De mystica theologia speculativa VI, 28 (éd. M. Vial, op. cit., p. 146-47).

66. Éd. P. Glorieux, t. II, p. 259-263. Voir A. Combes, La théologie mystique de Gerson. Profil de son évolution, Rome, Desclée, 1964, t. II, p. 569-668, repris et nuancé par M. Vial, Jean Gerson théoricien de la théologie mystique, Paris, Vrin, «Études de philosophie médiévale», 2006, p. 160 et suiv.

67. Éd. P. Glorieux, t. V, p. 531. 
et de silence préludant à la Pentecôte, à l'imitation des Apôtres au Cénacle. Les trois écritures - lyrique et effusive, exemplaire et oratoire, démonstrative et théorique - qui scandent cette progression spirituelle composent ainsi une «échelle céleste» que l'on peut rapprocher des trois «degrés» de la Montagne de contemplation. Or ce dernier texte figure dans les deux manuscrits qui conservent la Méditation: le rapprochement n'est donc pas fortuit; on évolue à l'intérieur du même paradigme, avec une unique visée, la pédagogie de la vie intérieure. D'une étape à l'autre, le style mime l'élévation, ou plutôt le désir qui la suscite et les résistances auxquelles elle se heurte. L'aboutissement réside dans une contemplation conçue comme extase intellectuelle, mais dont les effets se donnent à voir en termes de renovatio intérieure, par l'exercice des vertus et la pratique d'un «langage du ciel», celui-là même auquel nous convie par sa facture le discours gersonien.

isabelle.fabre@univ-montp3.fr 


\section{Annexe}

\section{Édition du texte}

Le texte de Gerson nous est transmis dans deux manuscrits conservés à la Bibliothèque nationale de France sous la cote fr. 990 (f. 235r-238v) et fr. 24839 (f. 66r-70r). Le premier, copié après 1427, était destiné à Jean II, duc de Bourbon, prince commanditaire d'œuvres originales de théologie, par ailleurs dédicataire du Livre du Cuer d'Amour espris (voir Léopold Delisle, Catalogue des manuscrits de la bibliothèque impériale, Paris, Imprimerie nationale, 1868, t. 1, p. 174, où le ms. fr. 990 est répertorié sous le titre de «Livre de contemplation»). Le deuxième a été acquis par Jean Lamasse, prieur de l'abbaye de Saint-Victor entre 1436 et 1449; c'est le $\mathrm{n}^{\circ} 17$ dans le catalogue établi par Danièle Calvot et Gilbert Ouy (L'œuvre de Gerson à Saint-Victor de Paris. Catalogue des manuscrits, Paris, Éditions du CNRS, 1990, p. 94-101). Il comporte une table signalant l'œuvre sous le titre de Devote meditationes anime circa ascensionem Christi. On en connaît aussi une traduction latine, qu'Ellies du Pin reproduit dans son édition des Opera omnia (1706) d'après le manuscrit de Saint-Victor (t. III, col. 697-699). On a choisi pour texte de référence le recueil de Jean de Bourbon (désigné par B dans l'apparat). Ce manuscrit sert de base à l'édition Glorieux (Jean Gerson, CEuvres complètes, Paris, Desclée \& Cie, 1966, t. VII, n 329, p. 390-393), mais la transcription qui en est donnée présente un certain nombre d'erreurs et omissions. Dans le manuscrit B, la décoration et la graphie sont soignées, les abréviations rares, la langue peu marquée. On a reporté les pieds de mouche qui structurent le texte dans le manuscrit, en introduisant au besoin nos propres alinéas et en numérotant les paragraphes. L'apparat critique enregistre les variantes morphologiques et lexicales du manuscrit de Saint-Victor (V), mais ne reproduit pas les variantes orthographiques (pour une vue complète de ce témoin, on se reportera à l'édition d'Edmond Vansteenberghe, "Quelques écrits inédits de Jean Gerson. XII. Méditation sur l'Ascension», Revue des Sciences Religieuses, t. XVI, 1936, p. 39-46); il comporte aussi, dans quelques cas difficiles, un bref commentaire grammatical. Les modifications apportées au texte de référence sont indiquées par des crochets droits (ajouts) et des parenthèses (formes fautives à supprimer); elles correspondent toujours, sauf précision contraire, à la leçon de notre autre témoin. 
[1] Qui me dourrai pennes et elles a la semblance d'une colombe? Si voleray et me reposeray ${ }^{68}$.

[2] Veez cy que mon Dieu, mon maistre et mon espoux s'en vole en hault, plus hault que les vens dessus toutes les nues, voir au dessus de tous les cieulx ; et je, povre ame [235 $]$ desolee, demeure en bas, trop bas sa jus en terre: comme ii l'ensuivray je? Je suis lasse orpheninne; je suis veve $\mathrm{e}^{\mathrm{iii}}$ desconfortee: qui sera mon tuteur? Qui me consolera? Apreste toy a voler, a ensuir ton amy. Tu, triste de cuer, reprens couraige, efforce toy!

[3] II Mais helas, que sont devenus mes plumes de sainctes pensees et mes deux elles, l'une de bon desir, l'autre de devot espoir? Elles sont esparses ça et la en maintes parties, par evagacion ${ }^{\text {iv }}$ de [mon cueur en] ${ }^{v}$ cures terriennes, et ${ }^{\mathrm{vi}}$ ce peu qui en est demouré, qui n'est point du tout desraciné, helas que me prouffite ${ }^{69}$ ? La tenant ${ }^{\mathrm{vii}}$ de charnalité corrumpue a tout englué; la pesanteur de mon corps et le grief fais de mon iniquité, $\mathrm{et}^{\text {viii }}$ me traient a terre et en [si] parfont [que] ${ }^{\mathrm{ix}}$ ne me puis aidier ne relever: comment voleray je ? Maudiz soient les traitres oyselleurs ${ }^{70}-$ le monde, la char et l'ennemy ${ }^{71}$ - qui ont tendus las et glut et roys et trabuchés partout ou je aloye, feust es prez, fust es champs, fust en la ville, fust en mon boyre, fust en mon mengier, fust en mon lit ou dehors! Et mal me suy gardee, si suis cheute en leurs laz, et telement suis mal menee! O quelle misere! Par avant ${ }^{\mathrm{x}}$ je ne sentoye riens de ses empeschemens, mais en cuidant franchement m'eslever, $\mathrm{j}$ ' ay ${ }^{\mathrm{xi}}$ trop apparceü quelle mescheance m'est avenue et ou je estoyexii boutee.

[4] II Si demande(s) maintenent elles nouvelles et plumes pures et nectes et entieres a la samblance de la $a^{\text {xiii }}$ colombe. Je ne vueil pas les plumes d'autrusse, par lesquelles je ne pourroye plus hault monter de terre: ce sont les plumes d'ypocrisie ${ }^{72}$. Je n'ay cure des plumes du ${ }^{\text {xiv }}$ faucon ou de l'autour, par lesquelles je ne pourroye repos trouver: ce sont [236 $]$ les plumes d'avarice et de rapacité; ainçoys je quiers les plumes de la

68. Ps. $54,7$.

69. Trad.: «... et les rares (plumes) qui me restent et ne m'ont pas été complètement arrachées, hélas, de quelle utilité me sont-elles ?»

70. Cf. Ps. 90, 3; 123, 7.

71. Thème des «trois ennemis de l'homme», fréquent dans la littérature morale à partir du $\mathrm{XII}^{\mathrm{e}}$ siècle, voir Pseudo-Bernard, Meditationes piissimae de cognitione humanae conditionis, XII (PL 184, col.503).

72. L'autruche n'a pas forcément mauvaise réputation dans les bestiaires, à l'exception notable du bestiaire Ashmole, qui l'assimile aux pharisiens ( Le Bestiaire: texte intégral en français moderne, reproduction en fac-similé des miniatures du Bestiaire Ashmole 1511 de la Bodleian Library d'Oxford, éd. Xénia Muratova et Daniel Poirion, trad. Marie-France Dupuis et Sylvain Louis, Paris, Ph. Lebaud, 1988, p. 117-118). 
colombe, les pennes de simplesse ${ }^{73}$ et de devote meditacion: si voleray et me reposeray.

[5] II Las, je ne puis! Mon Dieu, mon maistre ${ }^{\mathrm{xv}}$, mon pere de toute benignité, secourez moy ou descendez en bas a vostre povre fille consoler et relever, ou ${ }^{\text {xvi }}$ 'octroiez plumes et elles moyennant lesquelles je puisse a vous vouler en hault, bien hault jusques au lieu secret de vostre demeure es sains cieulx lassus, ou doit estre la conversation de toutes ames devotes ${ }^{74}$, la ou vous estes monté, vous qui estes leur amy loyal, le tout bel, le tout doulx, le tout bon, le tresglorieux espoux.

[6] II Que di je, mescheante, que desiré je, que souhaidé je ? Suis je digne de cest estat, de cest lieu, de cest honneur? Nennil voir, je le confesse ; mais, sire et pere de toute consolacion, vostre piteuse misericorde ${ }^{\text {xvi }} \mathrm{me}^{\mathrm{xvii}}$ donne le congié d'ainsy parler et desirer; neccessité tresamere me contraint et ${ }^{\text {xix }}$ vostre amour, l'amour de vostre presence ${ }^{\mathrm{xx}}$, de vostre beaulté et et ${ }^{\mathrm{xxi}}$ de vostre compaignie me fait souspirer; a vous me fait gemir ${ }^{\text {xxii }}$, plaindre et plourer, quant je me voy seulete desconfortee en ce present exil, en ceste vallee ${ }^{\text {xxiii }}$ obscure et tenebreuse $^{75}$, en ce desert horrible plain de toute adversité, de toute misere et infelicité. II Quelle merveille se je vueil moy esloigner en fuyant ${ }^{76}$, se je quiers demourer hors [du monde] en lieu secret? Et la vous atendré, qui me pouez sauver et delivrer de la paour et povreté de mon esperit et de la tempeste du monde, ou quel est iniquité et contradiction, labeur et injustice, usure et dolosité 77 partout.

[7] If Et tu, mon corps, qui souloyes estre mon amy par [236 v $]$ aucun temps sans moy empeschier a aler a Dieu, mais estoies d'un consentement avec moy en la voye et ou service de Dieu, tu mangoyes avec moy les doulces viandes des devos pleurs et des ${ }^{\mathrm{xxi}}$ gemissemens a Dieu; If la mort adviengne a ceulx qui te ont cecy fait, les faulx ennemis qui te font faire ceste guerre a moy, ce discort ${ }^{\mathrm{xx}}$ et ceste rebellion, en ce que tu quiers maintenant tes plaisirs charnelz contre ma voulenté et en vagant partout es occupacions vaines ou nuisibles ${ }^{\mathrm{xxv}}$ sans oïr mon contredit! II Racordons nous ensemble: trop mieulx nous estoit en nostre paix que en ceste division honteuse, que en ceste noyse et riote tresangoisseuse. Tu ne pues voler en hault, je le sçay bien, au moins jusques en la presence de mon amy, mais je te supply, ne me soyes point a destourbier! Sueffre que je recueille mes plumes de sainctes pensees et mes deux elles de desir et ${ }^{\mathrm{x} x v i \mathrm{i}}$ bon espoir; sueffre que je les nectoye et despesche ou que

73. Cf. Lc 3, 22.

74. Cf. Ph. 3, 20

75. Cf. Ps. $22,4$.

76. Cf. Ps. 54,8 .

77. Usure au sens d'«abus»; dolosité (emprunt savant au latin dolosus), «ruse». 
je en acquiere nouvelles: si m'en yray a mon amy; si voleray et me reposeray avec lui.

[8] Mon Dieu et mon espoux es[t] monté es cieulx moult ${ }^{\mathrm{xxviii}}$ joyeusement et tresglorieusement $^{78}$, avecques lequel monsterent tous les anciens prophetes $^{\mathrm{xxix}}$ et patriarches et autres justes. Presens estoit, n'est point doubté, la compaignie des anges, et sembleroit que tous les prisonniers qui lors estoient en purgatoire furent delivrez, comme a la joye nouvelle de la feste bien appartenoit; sembleroit aussi ${ }^{\mathrm{xxx}}$ que plusieurs y monterent a tout leurs corps gloriffiez, en especial ceulx qui ressusciterent aprés sa ${ }^{\mathrm{xxx}}$ passion $^{79}$. Et vous, Vierge tresdigne, que dites vous a celle heure, quant vous le veïstes partir? Que dirent tous les appostres et les diciples aussi? Mains doulx souspirs y ot, [237'] c'est bien a croire, mains regrez, mains souhaiz ${ }^{\mathrm{xxxii}}$ semblables aux miens mais trop plus grans, pour s'en vouler avec lui lassus, mesmement corporellement se son plaisir y eust esté. Neantmoins vous fistes ce que vous estoit loysible ${ }^{\text {xxiii }}$ et octroyé: vous y envoiastes chascun [1'] esperit par les pennes treslegieres de contemplacion, [puis que le corps] en bas y failloit demourer. II Les sains anges et archanges quelle feste firent ilz $^{\mathrm{xxxiv}}$, quelle melodie chanterent ${ }^{\mathrm{xxxv}}$ en regardant comment tous ceulx que Jhesucrist menoit de sa livree, ceulx de l'umain lignage, n'est point a recyter ne souffisamment penser. Lors fut ouverte la porte de paradis, qui par si long temps avoit esté close a tout l'umain ${ }^{\text {xxvi }}$ lignage; le ciel tout resplendit ${ }^{\text {xxxvii }} \mathrm{de}$ celle nouvelle venue, comme l'air reluist en la presence du soleil sans nuee. II En ceste volee en hault ne firent quelzconques empeschemens les ennemis d'umain lignage $\mathrm{e}^{80}$. O comme bien eureuse $\mathrm{e}^{\mathrm{xxxvii}}$ sera l'ame qui ainsi les eschevera ${ }^{\text {xxxix }}$, qui ainsi d'eulx (les) eschappera! Trop dure et trop hideuse et trop espouentable est leur encontre.

[9] II Jadis, comme on recite ${ }^{81}$, une devote personne, aprés ce qu'elle ot visité les sains lieux ou nostre sauveur Jhesucrist avoit esté corporellement, vint a la parfin ou mont ${ }^{\mathrm{xl}} \mathrm{d}^{\text {'Olivet }}{ }^{82}$. Illec ${ }^{\mathrm{xli}}$ adoura le lieu ou les benois

78. Cf. Ps. 46,6 .

79. D'après l'Évangile de Nicodème, XVII et suiv.

80. Cf. Pseudo-Augustin, Sermones suppositi de tempore, Sermo 179 In Ascensione Domini, IV, 2 (PL 39, col. 2085).

81. On reconnaît l'exemplum du «pèlerin de l'Ascension» (voir le Ci nous dit, éd. cit., p. 52 et Frederic C. Tubach, Index exemplorum, Helsinki, 1969, n 2497 et 3797). Thomas de Cantimpré fait aussi le récit d'une «mort de compassion» sur les lieux de la Passion (Bonum universale de apibus, I, 25, 5).

82. Lieu à partir duquel s'effectua l'Ascension d'après Lc 24, 50-51 (foras in Bethaniam). Une église (l'Imbomon) y fut construite par la suite; on y célébrait la fête dès le $\mathrm{IV}^{\mathrm{e}}$ siècle, le même jour que la Pentecôte, d'après le témoignage d'Égérie (Journal de voyage: itinéraire, éd. Pierre Maraval, Paris, Cerf, «Sources chrétiennes» 296, 1982, § 43, 4-5, p. 300-301). 
piés de Jhesus avoient esté, et apparoient les traces ${ }^{83}$. «Ou vous querray je plus, Sire? dit ceste devote personne; je ne sçay plus ou aler ce je ne vole a vous.» Lors elle esmut tant fort ses elles de saint desir que l'ame party du corps et s'en vola. O qui me pourra donner telles puissans eles ${ }^{\text {xlii }}$ ? Si voleray et me reposeray.

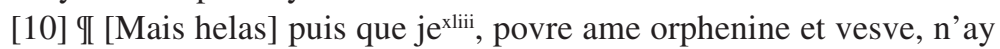
point ceste grace, ceste force et ce congié de m'en voler hors de mon corps $\left[237^{\vee}\right]$ avec mon doulx espoux, le vray amy de mon cuer, je feray au moins a l'exemple de Nostre Dame et des appostres ${ }^{84}$; car aprés ce que je seray alee par obeissance ${ }^{x l i v}$ sus le mont d'Olivet, le mont de doulce ${ }^{x l v}$ compassion a moy et aux autres, ou Jhesus commande ${ }^{x l v i}$ a aler, aprés ce que j'auray beu et mangié avec lui en la table du saint sacrement de l'autel, aprés ce qu'il aura reprouvéxlvii et argué mon incredulitée ${ }^{85}$, puis qu'il m'aura deffendu encquerir du temps futur par curiosité affin que tousjours je soye sur ma garde; en surplus, puis que je l'[auray] ${ }^{\text {xlvii }}$ veu monter en hault par la nue clere de vraye foy, puis que j'auray veu et oÿ les anges en guise ${ }^{x l i x}$ d'ommes de blanc (estoient) vestus, qui me certiffieront de son second ${ }^{1}$ advenement, que feray je? Je m'en retourneray a joye a ma conscience qui doit estre Jherusalem, la cité ou paix se doit veoir et trouver, et ou mont de Syon ${ }^{\text {li }}$, le mont de contemplacion ${ }^{86}$. La m'enfermeray je ou grant ${ }^{\text {lii }}$ senacle, ou senacle de charité large et espandue; je fermeray les portes de mes cinq sens pour la paour dez juifs ${ }^{87}$, c'est assavoir de tous mes ${ }^{\text {liii }}$ adversaires. Illec en seant et en priant je actendray jusques a tant que je soye revestue et [visitee] ${ }^{\text {liv }}$ de la vertus d'en hault du Saint [Espritt ${ }^{\text {tv }}$; j'actendray qu'il tonne si hault que je m'esmeuve toute ${ }^{\text {lvi }}$ par saincte paour et que ma sorde oreille soit ouverte a ce grant son; je actendray qu'il envoye feu si cler et si vif et si ardant que je soye toute enluminee, animee et embrasee, affermee ${ }^{\text {lvii }}$ contre tous maulx et aux louanges divines tresbien enlangagee.

[11] If Quant la saincte ame monte en la hautesse de son cuer par contemplacion, Dieu [238 ${ }^{r}$ l'eslieve ${ }^{\text {lviii }}$ en hault, selon le dit du Prophete ${ }^{88}$; car tant plus semble Dieu hault et incomprenable a icelle comme elle

83. Cf. Jacques de Voragine, Légende dorée, ch. 67 (éd. A. Boureau, Paris, Gallimard, «La Pléiade », p. 381) qui réfère à Sulpice Sévère (Chronique, II, 33) et à la Glose (glose marginale à Lc 22, 12, éd. cit., t. IV, p. 222a-b).

84. L'interprétation tropologique suit la trame d'Act. 1, 6-11.

85. Cf. Mc 16, 14.

86. Cf. Isidore de Séville, Étymologies, XV, I, 5: «Sion, quae Hebraice interpretatur speculatio... Hierusalem autem pacifica in nostro sermone transfertur» (éd. Lindsay, Oxford, 1911, t. 2, p. 149).

87. Cf. Jn 20, 19.

88. Éz. 11,1 
s'efforce plus de l'aprouchier. Mais ne s'en plaint point l'ame, ainçoys s'en tourne plus a l'onnor[er] ${ }^{\text {lix }}$, a le doubter et a mercier. Si est Dieu, qui ne puet estre comprins, [tant plus cognu comme on espreuve que il ne peust estre comprins $]^{1 \mathrm{x}}$ [ou tout $]^{1 \mathrm{xi}}$ cogneu, ainsi ${ }^{1 \mathrm{xii}}$ comme ung abisme sans fons donne congnoissance merveilleuse aux regardans, en ce quelexii plus y regarde on et moins on puet son fons veoir.

[12] II En ceste elevacion de terre en hault par contemplacion, l'ame trait tout a soy mesmes ${ }^{89}$, car n'est chose lors que l'ame voye ou oye ou sente ou gouste ou taste ou flaire ou pense que elle ne torne tout a son prouffit, a soy lever en hault pour Dieu mieulx assavourer, sentir et desirer. Par le contraire est de l'ame qui verse a terre scienment, car de tout fait son dommage; tout s'apesantist sur elle pour elle confondre et empeschier a relever.

[13] Creature humaine est un petit monde qui est raempliz de tant de gens comme dedans elle sont cogitacions et affeccions et pensees et ymaginacions et voulentés diverses. Jhesucrist doncques ${ }^{1 \mathrm{kiv}}$, quant ${ }^{\mathrm{lxv}}$ monte en hault dedans l'ame ${ }^{90}$ jusques au souverain ciel de l'esperit ${ }^{91}$, qui est la plus haulte partie de raison car elle touche a Dieu sans moyen, l'ame est envoyee preschier et denoncier la loy de l'Evangille a toutes gens pour les bien rigler et ordonner et pour les baptisier et laver en l'eaue de sainctes lermes, ou non du Pere et du Filz et du Sainct Esperit ${ }^{22}$. If Reçoyt en oultre l'ame telle puissance et telz signes ${ }^{93}$, car elle chace hors les [ $\left.238^{v}\right]$ ennemis par les exhorcismes d'oroisons; elle parle langage nouvel, langage du ciel; elle oste les serpens des venimeuses pensees; elle guarist toute enfermeté dedans soy par les mains de bonne operacion; et se elle boit ${ }^{\text {txvi }}$ aucun venin mortel par mauvaise suggescion ou temptacion, rien ne lui nuist a mort, car ce venin ne va point jusques au vif du cuer par mauvais consentement $\mathrm{et}^{\text {txvii }}$ deliberacion parfaicte.

Deo gratias ${ }^{\mathrm{Ixviii}}$.

89. Thème bonaventurien de la reductio de l'univers à Dieu par l'homme microcosme (cf. Itinerarium mentis in Deum, II, 1-4), souvent repris par Gerson. Voir en particulier son sermon pour le Jeudi Saint A Deo exivit (éd. P. Glorieux, t. V, n ${ }^{\circ} 208$, p. 14).

90. Lecture possible du Ps. 83, 6 (ascensiones in corde suo disposuit, litt. «il a disposé des montées dans son cœur»).

91. Il s'agit du coelum supremum ou intelligentia simplex, la plus haute faculté cognitive d'après Richard de Saint-Victor (Benjamin major, I, 9, PL 196, col. 74C), nomenclature reprise par Gerson dans son De mystica theologia speculativa, II, 10 (éd. A. Combes, op. cit., p. 28, trad. M. Vial: Jean Gerson, Sur la théologie mystique, op. cit., p. 80-81).

92. Cf. Matth. 28, 19.

93. Cf. Mc 16, 17-18 où le Christ énumère les signa qui accompagneront les disciples envoyés en mission. 
i. $\quad$ futur $p 3$ de «donner»

ii. comment $\mathrm{V}$

iii. $\quad$ "veuve» (la graphie veve ne note pas la labialisation de e central au contact de v à la fin du XII $s$.)

iv. l'evagacion $\mathrm{V}$, «distraction» (qui perturbe l'esprit et l'empêche de se fixer sur l'objet de sa méditation)

v. om. B

vi. en $\mathrm{V}$

vii. puantise rajouté en marge, tenant barré $\mathrm{V}$ : le tour expressifd'identification (type li fel d'anemi) formé de l'adjectif tenant, «collant, gluant», n'a manifestement pas été compris par le copiste

viii. et conjonction renforçant la corrélation entre les deux verbes avec une valeur d'insistance: "... non seulement m'attirent à terre, mais m'entraînent si bas que...»

ix. $\quad$ en parfont si ne $\mathrm{B}$; si rajouté en marge mais sans point d'insertion $\mathrm{V}$

x. par avant om. $\mathrm{V}$

xi. je i ay suscrit $\mathrm{V}$

xii. a. en quelle meschanseté je e. $\mathrm{V}$

xiii. une $\mathrm{V}$

xiv. d'un V

Xv. mon maistre om. $\mathrm{V}$

xvi. en $\mathrm{V}$

xvii. mere $\mathrm{V}$

xviii. m'en $\mathrm{V}$

xix. a V

xx. p. a vous me fayt gemir d. V

xxi. om. $\mathrm{V}$

xxii. a vous me fait gemir om. V

xxiii. vale $\mathrm{B}$

xxiv. des om. V

xxv. desconfort $\mathrm{V}$

xxvi. invisibles $\mathrm{BV}$, avec un jambage surnuméraire (leçon à écarter car elle introduit une incohérence, l'invisible étant du côté du spirituel, donc à l'opposé des occupacions vaines)

xxvii. et de b. V

xxviii. moult hault j. V

xxix. les prophetes anciens et $\mathrm{V}$

xxx. s'ainssy on lit $\mathrm{V}$

xxxi. la V

xxxii. soulas $\mathrm{V}$

xxxiii. possible $\mathrm{V}$

xxxiv. f. yl firent q. V

xxxv. c. il en $\mathrm{V}$

xxxvi. tout humain $1 . \mathrm{V}$

xxxvii. ciel resplendit tout de $\mathrm{V}$

xxxviii. enseure $\mathrm{V}$

xxxix. qui ainsi les eschevera $o m$. V (eschiver, «éviter»)

xl. moult V

xli. olivet et ilec $\mathrm{V}$

xlii. qui me donra teles pennes et ellez $\mathrm{V}$

xliii. moy V

xliv. obedience $\mathrm{V}$ 


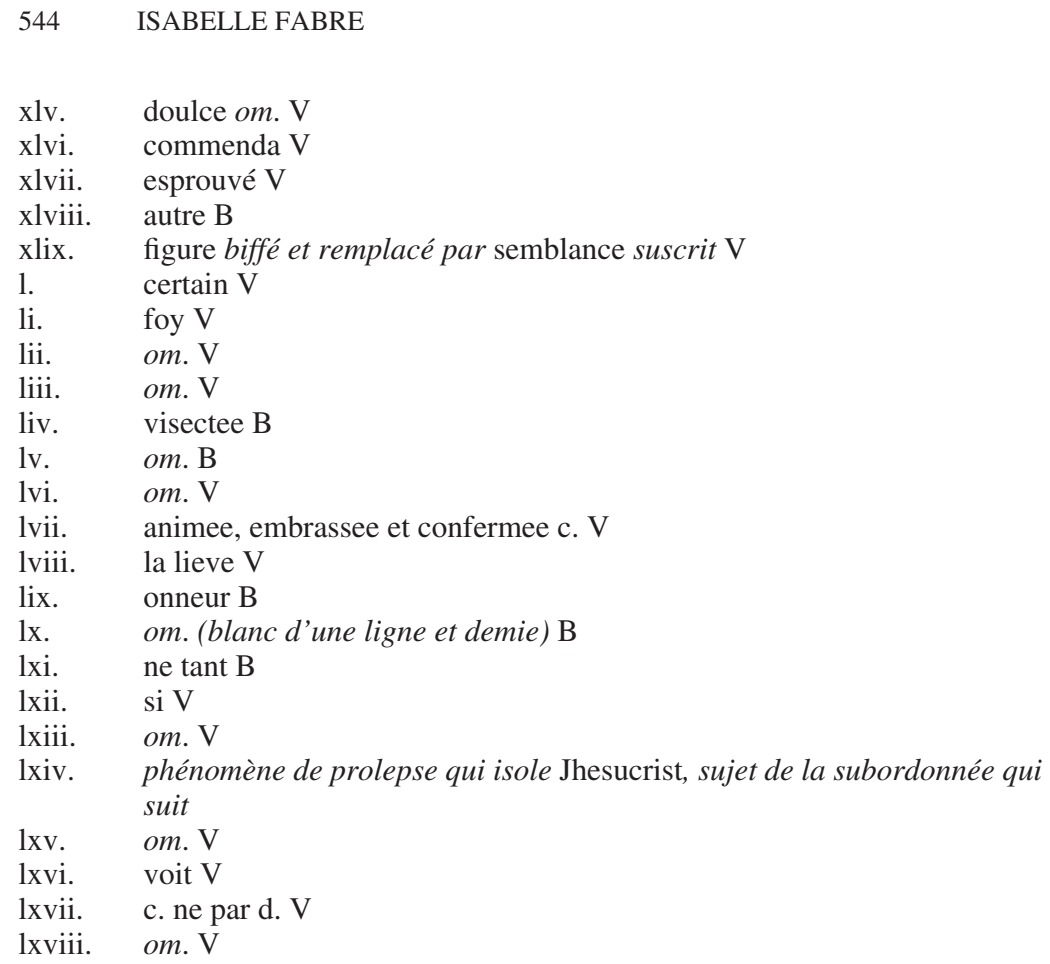

